\title{
ON CELLULAR AUTOMATON APPROACHES TO MODELING BIOLOGICAL CELLS
}

\author{
MARK S. ALBER*, MARIA A. KISKOWSKI ${ }^{\dagger}$, \\ JAMES A. GLAZIER ${ }^{\ddagger}$, AND YI JIANG ${ }^{\S}$
}

\begin{abstract}
We discuss two different types of Cellular Automata (CA): lattice-gasbased cellular automata (LGCA) and the cellular Potts model (CPM), and describe their applications in biological modeling.

LGCA were originally developed for modeling ideal gases and fluids. We describe several extensions of the classical LGCA model to self-driven biological cells. In particular, we review recent models for rippling in myxobacteria, cell aggregation, swarming, and limb bud formation. These LGCA-based models show the versatility of CA in modeling and their utility in addressing basic biological questions.

The CPM is a more sophisticated CA, which describes individual cells as extended objects of variable shape. We review various extensions to the original Potts model and describe their application to morphogenesis; the development of a complex spatial structure by a collection of cells. We focus on three phenomena: cell sorting in aggregates of embryonic chicken cells, morphological development of the slime mold Dictyostelium discoideum and avascular tumor growth. These models include intercellular and extracellular interactions, as well as cell growth and death.
\end{abstract}

1. Introduction. Cellular automata $(\mathrm{CA})$ consist of discrete agents or particles, which occupy some or all sites of a regular lattice. These particles have one or more internal state variables (which may be discrete or continuous) and a set of rules describing the evolution of their state and position (in older models, particles usually occupied all lattice sites, one particle per node, and did not move). Both the movement and change of state of particles depend on the current state of the particle and those of neighboring particles. Again, these rules may either be discrete or continuous (in the form of ordinary differential equations (ODEs)), deterministic or probabilistic. Often the evolution rules apply in steps, e.g., a motion or transport step followed by a state change or interaction step. Updating can be synchronous or stochastic (Monte-Carlo). At one extreme the rules may approximate well known continuous partial differential equations (PDEs), at the other they may resemble the discrete logical interactions of simple

*Department of Mathematics and Interdisciplinary Center for the Study of Biocomplexity, University of Notre Dame, Notre Dame, IN 46556-5670 (malber@nd.edu). Research partially supported by grant NSF IBN-0083653.

${ }^{\dagger}$ Department of Mathematics, University of Notre Dame, Notre Dame, IN 46556-5670 (mkiskows@nd.edu). Research partially supported by the Center for Applied Mathematics and the Interdisciplinary Center for the Study of Biocomplexity, University of Notre Dame, and by DOE under contract W-7405-ENG-36.

${ }^{\ddagger}$ Department of Physics and Biocomplexity Institute, Indiana University, Bloomington, IN 47405-7105 (glazier@indiana.edu). Research partially supported by grants NSF IBN-0083653, NSF INT98-02417, DOE DE-FGO299ER45785 and NASA NAG3-2366.

$\S$ Theoretical Division, Los Alamos National Laboratory, Los Alamos, NM 87545 (jiang @lanl.gov). Research supported by DOE under contract W-7405-ENG-36. 
Boolean computers [34]. Sophisticated flock models are an intermediate case of great current interest (e.g. $[86,136])$.

CA may produce very sophisticated self-organized structures. Von Neumann showed that a CA with a finite number of states and shortrange interactions could build a universal computer [154] and Conway in 'Life' demonstrated that even a simple two-state CA with purely local interactions could generate arbitrarily complex spatio-temporal patterns [50]. More recently, Wolfram has investigated the theory of CA and made a strong case for their utility in addressing complex problems [163-165].

This review illustrates CA approaches to biological complexity by describing specific biological models using two different types of cellular automata: lattice-gas-based cellular automata (LGCA-based) and the cellular Potts model (CPM).

One motivation for using cellular automata is the enormous range of length scales of typical biological phenomena. Organisms may contain dozens of organs composed of tissues containing tens of billions of cells. Cells in turn contain structures with length scales from Angstroms to several microns. To attempt to describe a cell in terms of individual molecular dynamics is hopeless. However, the natural mesoscopic length scale of a tissue is the cell, an autonomous agent with certain properties and certain responses to and effects on its surroundings. Since using the extreme simplification of a CA approach, which treats cells as simple interacting agents, we can simulate the interactions of tens of thousands to millions of cells, we have within reach the smaller-scale structures of tissues and organs that would be out of reach of more sophisticated (e.g., finite element) descriptions $[26,37]$. Nevertheless CA can be sophisticated enough that they can reproduce almost all commonly observed types of cell behavior. Ultimately, we hope to be able to unify, or at least cross-validate, the results of molecular dynamics, mesoscopic and continuum models.

Philosophically, CA are attractive because their large-scale behaviors are completely self-organized rather than arising from responses to externally imposed signals $[9,133]$. An individual cell has no sense of direction or position, nor can it carry a road map that tells it where to go (e.g., "one micron distal and two microns lateral"). It can only respond to signals in its local environment. Thus the traditional Wolpertian view of development via "Positional Coding" is untenable. Local environmental cues that can provide direction and location information may be self-organized or externally generated, with the cells responding passively to the signal. CA models favor self-organization while continuum PDE models generally (though not always) take a Wolpertian point of view. An added advantage of CA models is that they need not privilege any single cell as pacemaker or director - all cells are fundamentally equivalent.

We may view CA as discrete-time interacting ensembles of particles [34]. LGCA are relatively simple CA models, in which the particles select from a finite number of discrete allowed velocities (channels). During the 
interaction step particles appear, disappear or change their velocity state. During the transport step all particles simultaneously move in the direction of their velocity. LGCA can model a wide range of phenomena including the diffusion of ideal gases and fluids [70], reaction-diffusion processes [18] and population dynamics [111]. Dormann provides a wonderful introduction to CA [34]. For details about CA models in physics see Chopard and Droz [19] and specifically for lattice-gas models see Wolf-Gladrow [162] and Boon et al. [14]. In their biological applications LGCA treat cells as pointlike objects with an internal state but no spatial structure. The CPM is a more complex probabilistic CA with Monte-Carlo updating, in which a cell consists of a domain of lattice sites, thus describing cell volume and shape more realistically. This spatial realism is important when modeling interactions dependent on cell geometry. The original Potts model dates from 1952 [119] as a generalization of the Ising model to more than two spin states. It attracted intense research interest in the 1970s and 1980s because it has a much richer phase structure and critical behavior than the Ising model [116]. Glazier and Graner [53] generalized the Potts model to the CPM to study the sorting of biological cells. In the CPM, transition probabilities between site states depend on both the energies of site-site adhesive and cell-specific non-local interactions. The CPM represents different tissues as combinations of cells with different surface interaction energies and other properties. It describes other materials, like liquid medium, substrates and extracellular matrix (ECM) as generalized cells.

In this review we focus on modeling morphogenesis, the molding of living tissues during development, regeneration, wound healing, and various pathologies. During morphogenesis to produce body plans, organs and tumors, tissue masses may disperse, condense, fold, invert, lengthen or shorten. Embryos and tissues seem to obey rules differing from the physical rules we associate with the ordinary equilibrium statistical mechanics of materials: their forms seem to result from expression of intrinsic, highly complex, genetic programs. However, embryos, organs and healing and regenerating tissues assume many forms resembling those physics produces in non-living matter, suggesting that modeling based on physical mechanisms may be appropriate.

Biological cells interact with each other by two major means: local interaction by cell adhesion between cells in direct contact or between cells and their surrounding ECM, and longer range interactions such as signal transmission and reception mediated by a diffusing chemical field.

Cell adhesion is essential to multicellularity. Experimentally, a mixture of cells with different types and quantities of adhesion molecules on their surfaces will sort out into islands of more cohesive cells within lakes of their less cohesive neighbors. Eventually, through random cell movement, the islands coalesce [45]. The final patterns, according to Steinberg's Differential Adhesion Hypothesis (DAH) [142], correspond to the minimum of interfacial and surface energy. The DAH assumes that cell sorting re- 
sults entirely from random cell motility and quantitative differences in the adhesiveness of cells and that an aggregate of cells behaves like a mixture of immiscible fluids. In vitro $[11,46,47]$ and in vivo experiments $[54,56]$ have confirmed the soundness of the analogy. Moreover, cell adhesion molecules, e.g., cadherins (controlling cell-cell adhesion) and integrins (controlling cell-ECM adhesion), often serve as receptors to relay information to the cell [104] to control multiple cell-signaling pathways, including those of cell growth factors. Their expression and modification relate intimately to cell differentiation, cell mobility, cell growth and cell death (for reviews see $[51,97,143])$.

Chemotaxis is the motile response of cells or organisms to a gradient of a diffusible substance, either an external field or a field produced by the cells themselves. The latter is called chemotaxis signaling. Such non-local communication enables each cell to obtain information about its environment and to respond to the state of the cell community as a whole. In starved populations of Dictyostelium amoebae, some cells produce a communication chemical (cAMP), other active cells receive, produce and secrete the same chemical. The movement of Dictyostelium cells also changes from a random walk to a directed walk up the cAMP gradient. For sufficient densities of amoebae the signal induces cell aggregation to form a multi-cellular organism. Some bacteria broadcast a relayed stress signal that repels other mobile bacteria, which execute a biased random walk down the chemical gradient. In both cases the result protects the whole community from starvation. Unlike in differential adhesion, chemotactic cell motion is highly organized over a length scale significantly larger than the size of a single cell.

Both these interactions are essential to the biological phenomena described below. We demonstrate how LGCA and the CPM treat these interactions. Implementation of a CA model on a computer is straightforward. CA computations are numerically stable and are easy to modify by adding and removing local rules for state and position evolution. Ermentrout and Edelstein-Keshet [42] and Deutsch and Dormann [33] review some of the CA models that arise in simulations of excitable and oscillatory media, in developmental biology, in neurobiology and in population biology. We focus here on modeling aggregation and migration of biological cells. Both migration and aggregation occur in almost all organisms over a range of scales from sub-cellular molecular populations (e.g., actin filaments or collagen structures) to cellular populations (e.g., fibroblasts or myxobacteria to communities of organisms (e.g., animal herds or schools of fish) (see, amongst others $[10,21,63,99,118])$.

Advantages of CA include their simplicity, their ease of implementation, the ability to verify the relevance of physical mechanisms and the possibility of including relationships and behaviors which are difficult to formulate as continuum equations. In addition $\mathrm{CA}$ reflect the intrinsic 
individuality of cells. Limitations of CA include their lack of biological sophistication in aggregating subcellular behaviors, the difficulty of going from qualitative to quantitative simulations, the artificial constraints of lattice discretization and the lack of a simple mechanism for rigid body motion. In addition, interpreting simulation outcomes is not always as easy as for continuum equations.

2. LGCA models. This section illustrates several biological applications of LGCA models. We demonstrate the process of building LGCA models starting from a detailed description of a biological phenomenon and ending with a description of the results of numerical simulations.

2.1. Background of the LGCA model. In 1973 Hardy, de Passis and Pomeau [58] introduced models to describe the molecular dynamics of a classical lattice gas (hence "Hardy, Passis and Pomeau" (HPP) models). They designed these models to study ergodicity-related problems and to describe ideal fluids and gases in terms of abstract particles. Their model involved particles of only one type which moved on a square lattice and had four velocity states. Later models extended the HPP in various ways and became known as lattice gas cellular automata (LGCA). LGCA proved well suited to problems treating large numbers of uniformly interacting particles.

Like all CA, LGCA employ a regular, finite lattice and include a finite set of particle states, an interaction neighborhood and local rules which determine the particles' movements and transitions between states [34]. LGCA differ from traditional CA by assuming particle motion and an exclusion principle. The connectivity of the lattice fixes the number of allowed velocities for each particle. For example, a nearest-neighbor square lattice has four non-zero allowed velocities. The velocity specifies the direction and magnitude of movement, which may include zero velocity (rest). In a simple exclusion rule, only one particle may have each allowed velocity at each lattice site. Thus, a set of Boolean variables describes the occupation of each allowed particle state: occupied (1) or empty (0). Each lattice site can then contain from zero to five particles.

The transition rule of an LGCA has two steps. An interaction step updates the state of each particle at each lattice site. Particles may change velocity state and appear or disappear in any number of ways as long as they do not violate the exclusion principle. For example, the velocities of colliding particles may be deterministically updated, or the assignment may be random. In the transport step, cells move synchronously in the direction and by the distance specified by their velocity state. Synchronous transport prevents particle collisions which would violate the exclusion principle (other models define a collision resolution algorithm). LGCA models are specially constructed to allow parallel synchronous movement and updating of a large number of particles [34]. 
2.2. Applications of LGCA-based models in biology. Large groups of living elements often exhibit coordinated polarized movement. This polarization usually occurs via alignment, where individuals democratically align their direction and velocity with those of neighbors of the same type, rather than by aligning under the control of a single leader or pacemaker cell or in response to externally supplied cues [89, 90]. This selforganized local alignment admits multiple descriptions: for example, as an integro-differential equation as in Mogilner and Edelstein-Keshet [99, 100]. For an LGCA caricature of a simplified integro-differential model see Cook et al. [22]. Othmer et al. [112] describes a non-LGCA CA model for cell dispersion based on reaction and transport.

Many models of biological phenomena have employed PDEs to combine elements of random diffusive motion with biologically motivated rules that generate more ordered motion. These models, however, treat only local average densities of cells and do not include terms capturing the nonlocal interactions inherent in a population that moves as a collective unit. Nor do they include the discrete nature of cells and their non-trivial geometry and orientation. Mogilner and Edelstein-Keshet [99] and Mogilner et al. [100] realized that they could model such phenomena more realistically using integro-differential partial differential equations to account for the effects of "neighbor" interactions on each member of the population. In 1997, Cook et al. [22] described spatio-angular self-organization (the tendency of polarized cells to align to form chains or sheets) using an LGCA model based on a simplified integro-differential model.

Other manifestations of collective cell behavior are the several types of aggregation (see [34, 157] for details). For example, in differential growth, cells appear at points adjacent to the existing aggregate as described in [40] and [125]. In diffusion-limited aggregation (DLA) growing aggregates are adhesive and trap diffusing particles. Witten and Sanders [161] introduced DLA to model dendritic clustering in non-living materials, and Ben-Jacob and Shapiro $[133,134]$ have shown that DLA has extensive applications to bacterial colony growth in gels where nutrient or waste diffusion is slow (for more details see $[8,20,43,55,135]$.

Deutsch showed that although LGCA models like [16] and [29] which involve particles constantly moving with fixed velocities can model swarming, modeling aggregation requires resting channels.

A third mechanism for aggregation is chemotaxis by cells, either to a pacemaker or to a self-organized common center. If the cells secrete a chemoattractant, then a random fluctuation which increases local cell density will cause local chemoattractant concentration to increase, drawing in more cells and again increasing chemoattractant concentration in a positive feedback loop. Eventually the cells will all move into one or more compact clusters (depending on the range of diffusion of the chemoattractant and the response and sensitivity of the cells). 


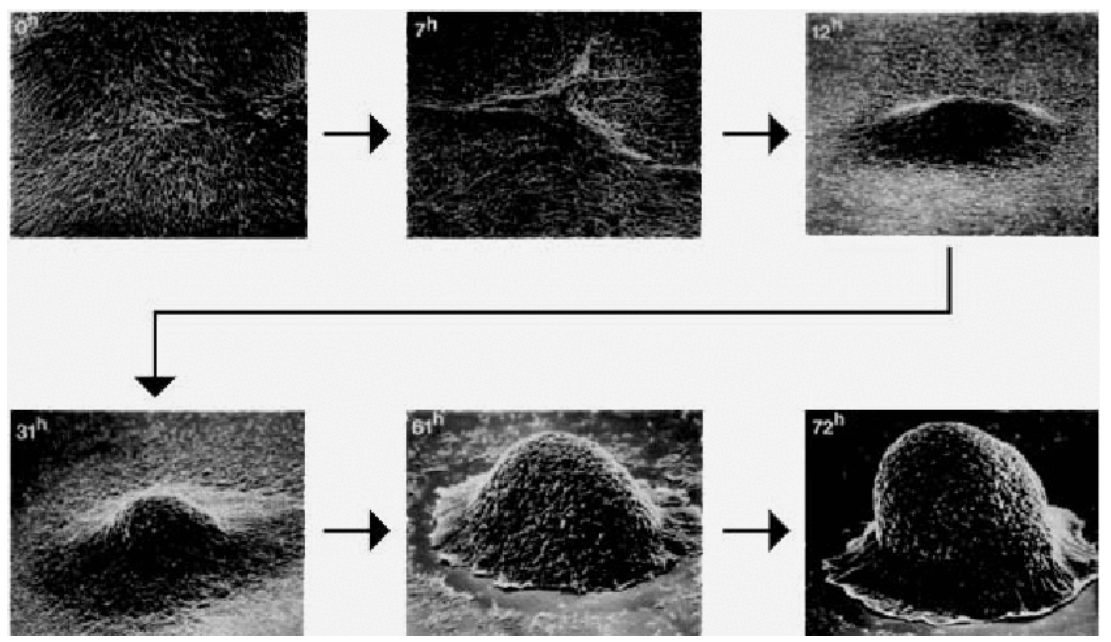

FIG. 1. Electron microscope image of fruiting body development in M. xanthus by J. Kuner. Development was initiated at 0 hours by replacing nutrient medium with a buffer devoid of a usable carbon or nitrogen source (from Kuner and Kaiser [80] with permission).

2.3. Rippling in myxobacteria. In many cases, changes in cell shape or cell-cell interactions appear to induce cell differentiation. For example, an ingrowing epithelial bud of the Wolfian duct triggers the formation of secretory tubules in the kidneys of mice [155] and in Dictyostelium pre-stalk cells sort and form a tip due to chemotaxis and differential adhesion [68]. The relationship between interactions and differentiation has motivated study of the collective motion of bacteria, which provides a convenient model for cell organization which precedes differentiation [9, 133]. A prime example is the formation of fruiting bodies in myxobacteria. Figure 1 illustrates fruiting body development in Myxococcus xanthus, which starts from starvation and undergoes a complex multi-step process of gliding, rippling and aggregation that culminates in the formation of a fruiting body with differentiation of highly polarized, motile cells into round, compact spores. A successful model exists for the more complex fruiting body formation of the eukaryotic Dictyostelium discoideum (see [68, 93]). Understanding the formation of fruiting bodies in myxobacteria, however, would provide additional insight since collective myxobacteria motion depends not on chemotaxis as in Dictyostelium but on mechanical, cell-cell interactions [39].

Rippling is a transient pattern that often occurs during the myxobacterial gliding phase before and during aggregation into fruiting bodies. During the gliding phase myxobacteria cells are very elongated, with a 10:1 length to width ratio, and glide over surfaces on slime tracks (see [166] 


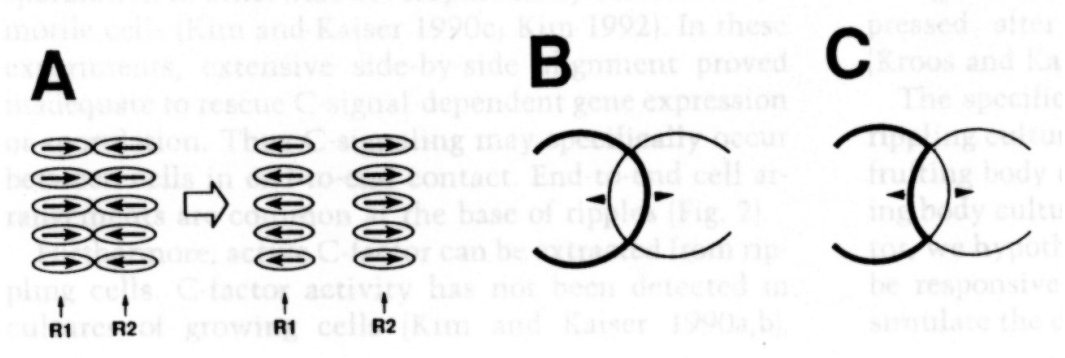

FIG. 2. (A) A reflection model for the interaction between individual cells in two counter-migrating ripple waves. Laterally aligned cells in counter-migrating ripples (labeled $R 1$ and R2) reverse upon end to end contact. Arrows represent the directions of cell movement. Relative cell positions are preserved. (B) Morphology of ripple waves after collision. Thick and thin lines represent rightward and leftward moving wave fronts, respectively. Arrows show direction of wave movement. (C) Reflection of the waves shown in B, with the ripple cell lineages modified to illustrate the effect of reversal. (From Sager and Kaiser [129] with permission.)

amongst others). The mechanism of cell motion is still not clear. Rippling myxobacteria form a pattern of equidistant ridges of high cell density that appear to travel periodically through the population. Tracking individual bacteria within a ripple has shown that cells oscillate back and forth and that each travels about one wavelength between reversals [129]. Cell movement in a ripple is approximately one-dimensional since the majority of cells move in parallel lines with or against the axis of wave propagation [129]. The ripple waves propagate with no net transport of cells and wave overlap causes neither constructive nor destructive interference [129].

Sager and Kaiser [129] have presented a model for myxobacterial rippling in which precise reflection explains the lack of interference between wave-fronts. Oriented collisions between cells initiate C-signaling which causes cell reversals. C-signaling occurs via the direct cell-cell transfer of a membrane-associated signaling protein (C-signal) when two elongated cells collide head to head. According to Sager and Kaiser's hypothesis of precise reflection, when two wave-fronts collide, the cells reflect one another, pair by pair, in a precise way that preserves the wave structure in mirror image. Figure 2 shows a schematic diagram of this reflection.

Current models for rippling (see $[15,63,90]$ ) assume precise reflection. Key differences among these models include their biological assumptions regarding the existence of an internal cell timer and the existence and duration of a refractory period during which the cell does not respond to external signals.

An internal timer is a hypothetical molecular cell clock which regulates the interval between reversals. The clock may speed up or slow down depending upon collisions, but each cell eventually will turn even without 
any collisions. An isolated cell oscillates spontaneously every 5-10 minutes with a variance in the period much smaller than the mean [63]. Also, observation of rippling bacteria reveals that cells oscillate even in ripple troughs where the density is too low for frequent collisions [160]. These observations both support an internal cell timer.

The refractory period is a period of time immediately following cell reversal during which cells are insensitive to $\mathrm{C}$-factor. The addition of exogenous C-factor up to a threshold value triples the reversal frequency of rippling cells [129]. Cells do not reverse more frequently at still higher levels of C-factor, however, suggesting the existence of a refractory period that sets a lower bound on the reversal period of a cell [129].

Although some evidence supports the role of both a refractory period and an internal cell timer in myxobacterial rippling, the question is still open. Igoshin et al. [63] describe a continuum model with both a refractory period and an internal cell timer which reproduces experimental rippling in detail. Börner et al. [15] reproduce ripples that resemble experiments, assuming a refractory period but no internal timer. Finally, Lutscher and Stevens [90] propose a one-dimensional continuum model which produces patterns that resemble ripples without invoking a refractory period or an internal timer.

We designed a fourth model for rippling to independently test both of these assumptions by including them separately in a simulation and comparing the simulations to experiments. Our LGCA model illustrates both the versatility of $\mathrm{CA}$ and their use to validate hypotheses concerning biological mechanisms.

Börner et al. [15] used an LGCA to model rippling assuming precise reflection and a cell refractory period, but no internal timer. Their temporally and spatially discrete model employs a fixed, nearest-neighbor square grid in the $x-y$-plane and an additional $z$-coordinate describing the number of cells that stack at a given lattice site. Particles have an orientation variable $\phi$ equal either to -1 or 1 corresponding to their gliding direction along the $x$-axis. Cells have a small probability $p$ of resting. Cells move along linear paths in the $x$-direction, so coupling in the $y$-direction is solely due to C-signal interaction.

At each time-step, particles selected at random move asynchronously one lattice site in the direction of their velocity vector. Each time-step of the model consists of one migration of all the particles and an interaction step. When a particle at height $z_{0}$ would move into a site that is already occupied at the same height, it has a $50 \%$ chance of slipping below or above the occupied position, adding another stochastic element to the model. A collision occurs for an oriented particle whenever it finds at least one oppositely oriented particle within a 5-node interaction neighborhood. The collision neighborhood extends the intrinsically one-dimensional cell movement to allow 2D rippling since the interaction neighborhood extends in the $y$-direction. 
If the cell is non-refractory, a single collision causes it to reverse. A cell reverses by changing the sign of its orientation variable.

Börner et al. [15] model the refractory period with a clock variable $\nu$ which is either 1 for a non-refractory cell or which counts $2, \ldots, r$ for $r$ refractory time-steps. A particle with a clock value 1 will remain in a nonrefractory state with value 1 until a velocity reversal, at which time the particle clock variable becomes 2 . During the refractory period, the clock variable increases by one unit per time-step until the clock variable is $r$. At the next time-step, the refractory period ends and the clock variable resets to 1 .

Starting from random initial conditions the model produces ripples which closely resemble experiment (compare [15], Figures 1(a) and 3(a)). The duration of the refractory period determines the ripple wavelength and reversal period. A refractory period of 5 minutes in the simulations reproduces experimental values for wavelength and reversal frequency. In the simulations, ripple wavelength increases with refractory period as in experiment [129]. Thus, the model shows that experiments are compatible with the hypotheses of precise reflection, a refractory period and no internal timer.

The LGCA we presented in [4] assumes precise reflection and investigates the roles of a cell refractory period and an internal cell timer independently. We model cell size and shape in an efficient way that conveniently extends to changing cell dimensions and the more complex interactions of fruiting body formation.

In experiments, cells do not reflect by exactly $180^{\circ}$ degrees. However, since most cells move roughly parallel to each other, models based on reflection are reasonable approximations. Modeling the experimental range of cell orientations would require a more sophisticated CA since LGCA require a regular lattice which does not permit many angles. Tracking of rippling cells (e.g., [128], Figure 6) seems to indicate that cells most often turn about $150^{\circ}$ degrees rather than $180^{\circ}$ degrees, which may be modeled using a triangular lattice (see Alber et al. [4]).

Our model employs a nearest-neighbor square lattice with three allowed velocities including unit velocities in the positive and negative $x$ directions and zero velocity. At each time-step cells move synchronously one node in the direction of their velocity. Separate velocity states at each node ensure that more than one cell never occupies a single channel.

We represent cells in our model as (1) a single node which corresponds to the position of the cell's center of mass in the $x y$ plane, (2) the choice of occupied channel at the cell's position designating the cell's orientation and (3) an interaction neighborhood determined by the physical size of the cell. We define the interaction neighborhood as an elongated rectangle to reflect the typical $1 \times 10$ proportions of rippling myxobacteria cells [129]. Oblique cells would also need an angle to designate their angle from horizontal. Representing a cell as an oriented point with an associated in- 
teraction neighborhood is computationally efficient, yet approximates continuum dynamics more closely than assuming point-like cells, since cell interaction neighborhoods may overlap in a number of ways. Several overlapping interaction neighborhoods correspond to several cells stacked on top of each other.

In our model, collisions occur between oppositely-oriented cells. A cell collides with all oppositely-oriented cells whose interaction neighborhoods overlap its own interaction neighborhood. Thus, a cell may collide simultaneously with multiple cells.

We model the refractory period and internal cell timer with three parameters; $R, t$ and $\tau$. $R$ is the number of refractory time-steps, $t$ is the minimum number of time-steps until a reversal and $\tau$ is the maximum number of time-steps until a reversal. Setting the refractory period equal to one time-step is the off-switch for the refractory-period and setting $\tau$ (the maximum number of time-steps until a reversal) greater than the number of time-steps of the simulation is the off-switch for the internal cell timer.

Our internal timer extends the timer in Igoshin et al. [63]. We borrow a phase variable $\phi$ to model an oscillating cycle of movement in one direction followed by a reversal and movement in the opposite direction. Thus, reversals are triggered by the evolution of this timer rather than directly by collisions as in the model of Börner et al. [15]. $0 \leq \phi(t) \leq \tau$ specifies the state of the internal timer. $\phi$ progresses at a fixed rate of one unit per time-step for $R$ refractory time-steps, and then progresses at a rate, $\omega$, that depends non-linearly on the number of collisions $n_{\mp}$ to the power $p$ :

$$
\omega_{ \pm}(x, \phi, n, q)=1+\left(\frac{\tau-t}{t-R}\right) *\left(\frac{\left[\min \left(n_{\mp}, q\right)\right]^{p}}{q^{p}}\right) * F(\phi),
$$

where,

$$
F(\phi)= \begin{cases}0, & \text { for } 0 \leq \phi \leq R \\ 0, & \text { for } \pi \leq \phi \leq(\pi+R) \\ 1, & \text { otherwise }\end{cases}
$$

This equation is the simplest which produces an oscillation period of $\tau$ when no collisions occur, a refractory period of $R$ time-steps in which the phase velocity is one, and a minimum oscillation period of $t$ when a threshold (quorum) number $q$ of collisions, $n_{c}$, occurs at every time-step. We assume quorum sensing such that the clock velocity is maximal whenever the number of collisions at a time-step exceeds the quorum value $q$. A particle will oscillate with the minimum oscillation period only if it reaches a threshold number of collisions during each non-refractory time-step (for $t-R$ time-steps). If the collision rate is below the threshold, the clock phase velocity slows. As the number of collisions increases from 0 to $q$, the phase velocity increases non-linearly as $q$ to the power $p$. 


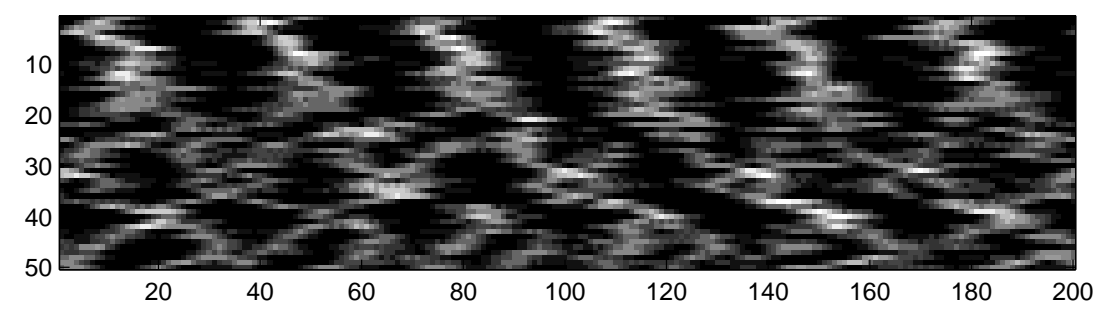

FIG. 3. Typical ripple pattern for myxobacteria simulations including both a cell clock and a refractory period. (Cell length $=5, \delta=2, R=10, t=15, \tau=25$.) Figure shows the density of cells (darker gray indicates higher density) on a 50x200 lattice after 1000 timesteps. (From Alber et al. [4].)

Results of numerical simulations. Our model forms a stable ripple pattern from a homogeneous initial distribution for a wide range of parameters, with the ripples apparently differing only in length scale (see Figure 3). Currently we are working to establish criteria for quantitative comparison of ripple patterns.

In our simulations the refractory period is only critical at high densities. Ripples form without an internal timer over the full range of ripple densities. Our model is most sensitive to the minimum oscillation time $t$, as ripples form only when $t$ is about 1 to 1.5 times larger than the refractory period.

The wavelength of the ripples depends on both the duration of the refractory period and the density of signaling cells. The wavelength increases with increasing refractory period (see Figure 4) and decreases with increasing density (see Figure 5).

Effect of dilution with non-signaling cells. Sager and Kaiser [129] diluted C-signaling (wild-type) cells with non-signaling ( $\operatorname{csg} \mathrm{A}$ minus) cells that were able to respond to $\mathrm{C}$-signal but not produce it themselves. When a collision occurs between a signaling and a non-signaling cell, the nonsignaling cell perceives C-signal (and the collision), whereas the C-signaling cell does not receive $\mathrm{C}$-signal and behaves as though it had not collided. The ripple wavelength increases with increasing dilution by non-C-signaling cells. Simulations of this experiment with and without the internal timer give very different results, see Figure 6 . The dependence of wavelength on the fraction of wild type cells resembles the experimental curve (see [129], Figure 7G) only with the internal timer turned off.

Since the wavelength decreases with increasing density, we ask if the wavelength of ripples in a population of wild type cells diluted with nonsignaling cells is the same as for the identical subpopulation of wild type cells in the absence of the mutant cells. Figure 7 shows the wavelength dependence on the density of signaling cells when only signaling cells are present (dotted line) and for a mixed population of signaling cells of the 
CELLULAR AUTOMATON APPROACHES TO BIOLOGICAL MODELING 1

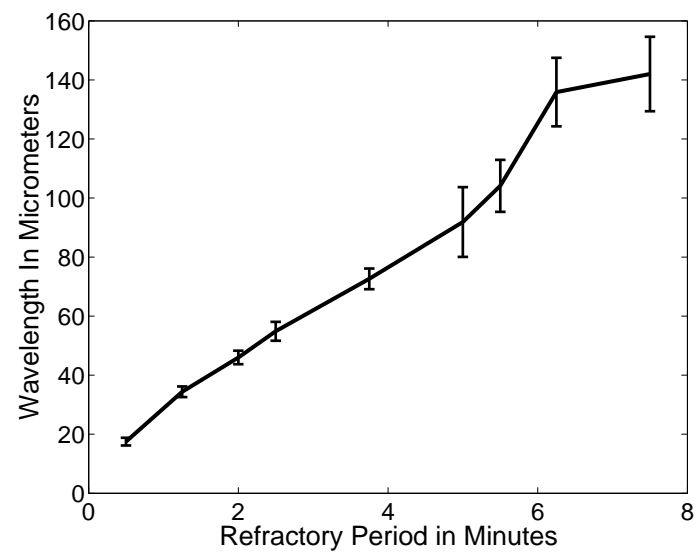

FIG. 4. Average wavelength in micrometers versus refractory period in minutes for myxobacteria simulations. Cell length $=4, \delta=1$ with the internal timer adjusted for each value of the refractory period $R$ so that the fraction of clock time spent in the refractory period is constant for each simulation: $t=3 R / 2$ and $\tau=5 * R / 2$. (From Alber et al. [4].)

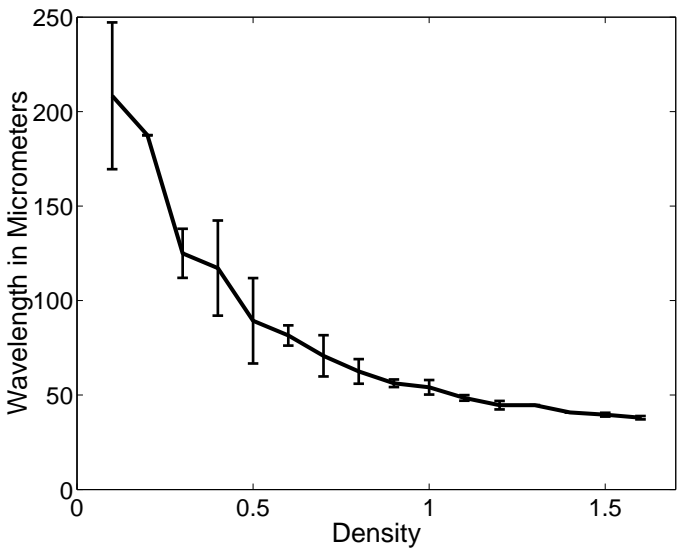

FIG. 5. Average wavelength in micrometers versus density for myxobacteria simulations (total cell area over total lattice area). Cell length $=4$ with an internal timer given by $R=8, t=12, \tau=20$. (From Alber et al. [4].)

same density with non-signaling cells (solid line). Apparently, the decrease in C-signal explains the increase in wavelength. The non-signaling mutants do not affect the pattern at all.

2.4. Cell alignment. Cook et al. [22] implemented an LGCA and reproduced the basic types of spatio-angular self-organization of a simplified version of the integro-differential models of Mogilner et al. [100]. In 


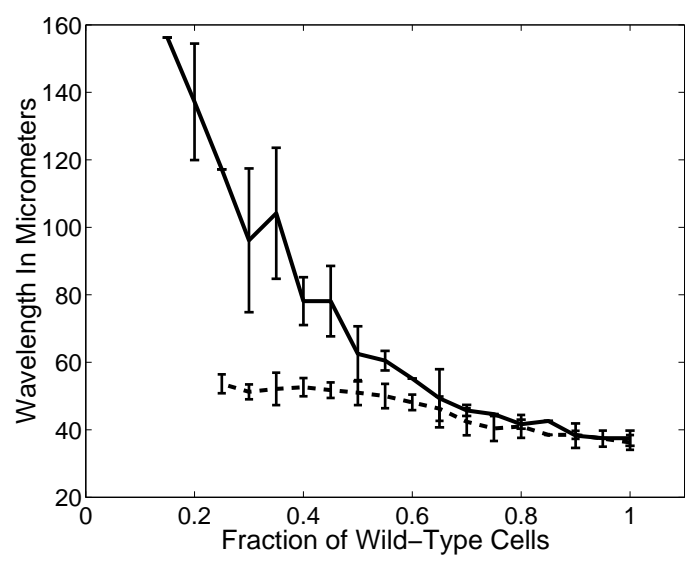

FIG. 6. Wavelength in micrometers versus the fraction of wild-type cells, in the presence (dotted line, $\tau=20$ ) and absence (solid line, $\tau=2000$ ) of an internal cell timer for myxobacteria simulations. Cell length=4, $R=8, t=12$. (From Alber et al. [4].)

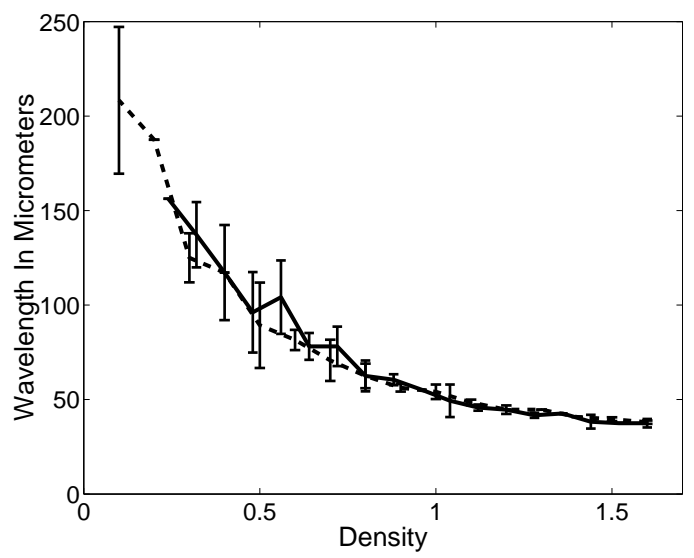

FIG. 7. Wavelength versus density with no internal timer $(\tau=2000)$ for myxobacteria simulations. Density is total cell area over total lattice area. The dotted line is the wavelength in micrometers versus the density of wild-type cells with no csgA-minus cells present. The solid line is the wavelength in micrometers versus the density of wild type cells when the density of csgA-minus cells is increased so that the total cell density remains 1.6. Cell length=4, $R=8, t=12, \tau=2000$. (From Alber et al. [4].)

their model each particle corresponds to one cell, the number of cells is fixed and automaton rules model the non-local character of the integrodifferential equations.

Deutch $[30,31]$ generalized this model by introducing different types of operators defined on orientation vectors at each lattice site and local 
orientation fields (see below for details). He showed that a simple dot product favors cell alignment. In these single cell-type models, clusters of cells with one preferred orientation grow and multiple clusters with the same orientation merge into a single large cluster.

Alber and Kiskowski [3] modeled the spatio-angular movement and interaction of $n$ types of cell. In this model cell behavior results from competition between two types of aggregation. In accordance with transitional probabilities, a cell can either align with the directional field of its neighbors or with other cells of its own alignment with a probability weighted by the neighborhood density of its own cell type. In the CA model we describe below, the clusters formed are confluent collections of particles of the same type moving in the same direction.

We describe in detail below a CA model for aggregation of aligned particles of $k$ different types. Consider $m$ square $(n \times n)$ lattices with nodes $\vec{r}$ and with periodic boundary conditions. Define state space stochastic Boolean variables,

$$
S^{(k)}=\left(s_{1}^{(k)}, s_{2}^{(k)}, s_{3}^{(k)}, s_{4}^{(k)}\right), \quad k=1, \ldots, m,
$$

where $s_{i}^{(k)}=1(0)$ indicate one of the four directions in the lattice and

$$
\eta^{(k)}(\vec{r})=\left(\eta_{1}^{(k)}(\vec{r}), \eta_{2}^{(k)}(\vec{r}), \eta_{3}^{(k)}(\vec{r}), \eta_{4}^{(k)}(\vec{r})\right), \quad k=1, \ldots, m,
$$

denote configurations at node $\vec{r}$ in the $m$ lattices. We impose an exclusion principle by limiting the sum of a node's densities to 4 :

$$
\rho(\vec{r})=\sum_{k=1}^{m} \rho^{(k)}(\vec{r})=\sum_{k=1}^{m} \sum_{i=1}^{4} \eta_{i}^{(k)}(\vec{r}) \leq 4 .
$$

By applying a template, we can describe the nearest neighbors to the node $\vec{r}$ of type $k$ as:

$$
N^{(k)}(\vec{r})=\left(r+c_{1}, r+c_{2}, r+c_{3}, r+c_{4}\right)
$$

where:

$$
c_{1}=(1,0), c_{2}=(0,1), c_{3}=(-1,0), c_{4}=(0,-1) .
$$

Then the local orientation fields are:

$$
O_{N(r)}^{(k)}=\sum_{i=1}^{4} \eta^{(k)}\left(r+c_{i}\right), \quad k=1, \ldots, m .
$$

We can also calculate local densities of particles of particular type $k$ simply by summing up the number of particles of this type which are nearest neighbors to a given node $\vec{r}$ :

$$
D_{N(\vec{r})}^{(k)}=\sum_{i=1}^{4} \rho^{(k)}\left(r+c_{i}\right) .
$$


Initially particles are randomly distributed on the lattice. Then we apply interaction and transport steps to every node in the lattice simultaneously.

The interaction obeys the following transition probabilities:

$$
A_{s, s^{\prime}}\left(O_{N(\vec{r})}^{(k)}\right)=\left\{\begin{array}{l}
\frac{M\left(s^{\prime}\right)}{Z(s)} \text { if } \quad \rho\left(s^{\prime}\right)=\rho(s) \\
0 \quad \text { else }
\end{array}\right.
$$

where

$$
M\left(s^{\prime}\right)=e^{\sum_{k=1,2}\left(\beta_{k}\left(O_{N(\vec{r})}^{(k)} \otimes s^{\prime}\right)+\gamma_{k} \rho^{(k)}(\vec{r})\left(D_{N(\vec{r})}^{(k)}\right)\right.},
$$

the normalization factor $Z(s)$ is chosen such that

$$
\sum_{s^{\prime}, \rho\left(s^{\prime}\right)=\rho(s)} A_{s, s^{\prime}}\left(O_{N(r)}^{(k)}\right)=1
$$

and

$$
O_{N(r)}^{(k)} \bigotimes s^{\prime}: N_{0}^{5} \rightarrow N_{0}
$$

is a bilinear functional. Choosing $O_{N(\vec{r})}^{(k)} \otimes s^{\prime}: \equiv<O_{N(\vec{r})}^{(k)}, s^{\prime}>$ favors parallel orientation. For details about different functionals see [30, 31]. If $O_{N(r)}^{(k)}=0$ a random discrete walk results.

We implement transport as follows: Particles move along their directions to their nearest neighbors:

$$
\eta_{i}^{(k)}(\vec{r}) \rightarrow\left(\eta_{i}^{(k)}(\vec{r})\right)^{T}:=\eta_{i}^{(k)}\left(r-c_{i}\right) .
$$

2.5. Gliding and aggregation in myxobacteria. During the aggregation phase of Myxococcus xanthus, cells stream towards aggregation centers to produce mounds of $10^{5}$ to $10^{6}$ cells. Highly elongated cells form chains or streams which spiral in to the aggregation centers [109]. The aggregation centers begin as small, asymmetric mounds which may diffuse or coalesce with other aggregation centers. As an aggregation center matures into a fruiting body, cells differentiate into non-motile round spores. The organization of cells within a fruiting body may reveal clues about aggregation center formation. Cells outside the periphery of a fruiting body form a spiral [38]; cells at the periphery pack tightly with their long axes parallel to the mound circumference, while cells in the mound center are less dense and less organized in arrangement [128].

$\mathrm{C}$-signal, a membrane-associated signaling protein, induces aggregation [65] and is required for normal aggregate formation [71]. Levels of Csignal are much higher than during rippling. Repeated efforts have failed to find a diffusing chemoattractant which could explain aggregation though chemotaxis. Observations of streams of cells passing a nearby aggregation 
center towards a center further away also discourage any chemotaxis-based aggregation model [65]. The passing cells do not displace towards the nearby mound as they would if they moved up a gradient of a diffusing chemical. Instead, they continue as if the second aggregation center were not present. Thus, aggregation appears to organize solely through cellcontact interactions.

Stevens' stochastic CA model of gliding and aggregation in myxobacteria employs self-attracting reinforced random walks and chemotaxis [144] to model bacteria, slime and a diffusing chemoattractant on a $100 \times 100$ nearest-neighbor square lattice with periodic boundary conditions. Her results provide an excellent example of how CA models can be used experimentally to test the validity and necessity of different parameters and assumptions.

Model bacteria are rod-shaped objects of eight nodes with one labeled pole node indicating the front of the cell. The cells are initially randomly distributed in the lattice and glide by moving their labeled front pole node into one of the three adjacent neighbors not already occupied by the cell's body. Cells glide preferentially on slime trails, glide faster on slime trails, glide preferentially towards the diffusing chemoattractant and keep their direction of motion without turning for about one cell length when neither slime nor chemoattractant influences their direction. The interaction neighborhood of a cell is the four nearest neighbors of the cell pole. A cell crossing a slime trail at an angle will reorient to follow the trail.

Bacteria deposit slime underneath their bodies at a rate $\kappa_{S}$. Slime decays at a rate $\lambda_{S}$. When cell density exceeds a critical value at a point under the area of a cell, cells produce chemoattractant at a rate $\lambda_{C}$. Chemoattractant decays at a rate $\kappa_{C}$.

Stevens used her cellular automata to test the hypothesis that a selfattracting reinforced random walk alone could account for aggregation in myxobacteria and tested the effects of several parameters: increasing the preference for gliding straight ahead, increasing slime production, and increasing the gliding velocity of cells traveling on slime trails. Additionally, she added cell-cell adhesion to the model to test the effect of cells preferring to glide parallel to their neighbors. She modeled adhesion as an enveloping, oriented structure to which adjacent bacteria have a high probability of aligning. Cell adhesion is uniform over the cell surfaces but the cell elongation encourages alignment.

Stevens found that self-attracting reinforced random walkers alone (with cells depositing slime, gliding preferentially on slime tracks and gliding faster on slime tracks) could not form stable aggregation centers. Unstable pre-aggregation patterns did form, however, that resembled experimental observations. Aggregates would form, diffuse away and reappear in other regions. Stable centers required an extra factor. For example, adding a diffusing chemoattractant stabilized the centers. Stevens speculated that a membrane-bound chemoattractant might also function as an attractive signal. 
Experimentally, cells glide faster on slime trails. Stevens modeled an increase in gliding speed on slime trails which produced larger aggregates in the model. Cell-cell adhesion caused cells to assemble in long chains.

2.6. Swarming. Swarming and flocking are a class of collective selforganization that emerges from a multitude of simultaneous local actions rather than following a global guide [9]. Swarming occurs in a wide variety of fields, including animal aggregation [114], traffic patterns [120], bacteria colonies [27], social amoebae cell migration [86], fish or bird flocking [9, 115] and insect swarming [138]. Swarming patterns all share one feature: the apparent haphazard autonomous activities of a large number of "particles" (organisms or cells), on a larger scale, reveal a remarkable unity of organization, usually including synchronized non-colliding, aligned and aggregate motion. Most models, however, only measure the density distribution, i.e. they look for nearly constant density in the center of the swarm and an abrupt density drop to zero at the edge [86, 100].

Many artificial-life simulations produce strikingly similar "emergent" characteristics, e.g., [13]. One such example is boids [124], simulated birdlike agents, where simple local rules such as 1) Collision Avoidance: avoid collisions with nearby flockmates, 2) Velocity Matching: attempt to match velocity with nearby flockmates, and 3) Flock Centering: attempt to stay close to nearby flockmates; give rise to complex global behaviors.

Most swarming models are of molecular dynamics type, with all particles obeying the same equations of motion and residing in a continuum rather than a lattice $[27,86]$. Multiple species may be present (e.g., [3]) but the properties of all members of a single species are identical. Particles have no "memory" of their behavior except for their current velocity and orientation. Particles are "self-propelled" [24, 153] since they move spontaneously without external forces, unlike non-living classical particles whose motions results from external forces. Some models require a nonlocal interaction, e.g., in the continuum model of Mogilner et al. [100], where integro-differential partial differential equations represent the effects of "neighbor" interactions, and in the particle model of Ben-Jacob et al. [7], in which a rotational chemotaxis field guides the particles. Recently Levine et al. [86] coarse-grained their particle model, which has only local interactions, to produce a continuum model and showed that the two models agree well with each other.

Unfortunately, either because we do not understand the interactions between particles well enough, or because their actions may depend in a complex way on the internal states and history of the particles, we cannot always describe particle interactions by an interaction potential or force. Phenomenological rules are then more appropriate. In such cases CA models are perfect for studying swarming as a collective behavior arising from individual local rules. 
Deutsch [29, 32] modeled examples of social pattern formation as LGCA based on the concept of "direct information exchange." Particles (cells, organisms) have some orientation, and can evaluate the orientations of resting particles within a given "region of perception." Simulations exhibit transitions from random movement to collective motion and from swarming to aggregation. Adamatzky and Holland [2] modeled swarming with excitable mobile cells on a lattice. By varying the duration of cell excitation and the distances over which cells interact and excite one another, they established many parallels with phenomena in excitable media.

2.7. Cluster formation by limb bud mesenchymal cells. Over 36-72 hours in a controlled experiment, a homogeneously distributed population of undifferentiated limb bud mesenchymal cells cluster into dense islands, or "condensations," of aggregated cells [83]. The condensations develop concurrently with increases in extracellular concentrations of a cellsecreted protein, fibronectin, a non-diffusing extracellular matrix macromolecule which binds adhesively to cell surface molecules, including receptors known as integrins, which can transduce signals intracellularly. The limb cells also produce the diffusible protein $\mathrm{TGF}-\beta$, which positively regulates its own production as well as that of fibronectin [79].

The roughly equally spaced patches of approximately uniform size are reminiscent of the patterns produced by the classical Turing reactiondiffusion mechanism. A Turing pattern is the spatially heterogeneous pattern of chemical concentrations created by the coupling of a reaction process with diffusion. In 1952, Alan Turing showed that chemical peaks will occur in a system with both an autocatalytic component (an activator) and a faster-diffusing inhibiting component (an inhibitor) [148]. Fluctuations of concentration of a particular wavelength grow while other wavelengths die out. The diffusion coefficients of the two components and their reaction kinetics, and not the domain size, determine the maximally growing wavelength [34]. For details about Turing pattern formation, see $[34,35,41,91,105]$. For details about the suggested role of reaction diffusion in the development of the vertebrate limb, see $[107,108]$.

Kiskowski et al. [79] model the production of fibronectin and subsequent limb bud patch formation using an LGCA-based reaction-diffusion process having TGF- $\beta$ as the activator but with an unknown inhibitor. In their model, cells are points that diffuse in a random walk on a nearestneighbor square lattice. At each time-step, cells choose either one of four direction vectors with equal probability, $p$, or a resting state with probability $1-4 p$. A higher probability of resting models slower diffusion. A cell-driven reaction-diffusion occurs between two chemicals (an activator, the morphogen $A$, and a faster diffusing inhibitor, the morphogen $B$ ) which diffuse and decay on the lattice. The production of activator and inhibitor occurs at lattice sites occupied by cells, while inhibition and diffusion occur throughout the lattice. The binding of cells to fibronectin results in slower 
diffusion, which we model by increasing the probability of assigning cells to resting states. When local levels of activator exceed a threshold, cells respond by secreting fibronectin to which they bind, reducing $p$ and causing clustering. All cells have fibronectin receptors and cells do not adhere to each other, but only to fibronectin molecules.

During each time-step, we model activation and inhibition as follows: Cells secrete a small basal amount of activator, increasing activator levels. Activator levels stimulate cells to produce more activator and inhibitor. Inhibitor levels decrease activator levels without requiring the presence of cells.

This relation between reaction and diffusion produces sharp peaks in concentration of both chemicals for specific parameter values by the classical Turing mechanism. The key parameters are $\delta_{A}$ and $\delta_{B}$, the diffusion rates of morphogens $A$ and $B$, the activation rates of activator and inhibitor $\alpha_{A}$ and $\alpha_{B}$, the inhibition rate of activator $\beta_{A}$ and the maximum rates at which a cell can produce morphogens $A$ and $B, A_{\max }$ and $B_{\max }$. Increasing the diffusion rate of morphogen $A$ widens the peaks and increasing $\alpha_{B}$ increases the distance between peaks. Adjusting these parameters allows us to reproduce patch formation qualitatively similar to experiments [79].

Although this model makes many simplifications (cells are points, parameter values are arbitrarily chosen) it does show that cell-driven reactiondiffusion may create strong chemical peaks in morphogen levels and that for rather simple assumptions, fibronectin clusters can be expected to colocalize with morphogen peaks in the form of islands [79]. The model may also yield insight into the causes of variations in condensation (e.g., along the proximo-distal axis of the limb or between forelimb and hindlimb) since simulation results have shown that increasing $\alpha_{B}$ increases the distance between peaks and increasing the diffusion rate of morphogen $A$ broadens the peaks.

3. The cellular Potts model. LGCA models are convenient and efficient for reproducing qualitative patterning in bacteria colonies, where cells retain simple shapes during migration. Eukaryotic cells such as amoebae, on the other hand, move by changing their shapes dramatically using their cytoskeleton. In many circumstances, we can treat cells as points on a lattice despite their complex shapes. In other cases, such as the sporulation of myxobacteria, where the cells differentiate from rod-shaped into round spore cells, shape change may be responsible for the patterning, hence requiring a model that includes cell shape. The CPM is a flexible and powerful way to model cellular patterns that result from competition between a minimization of some generalized functional of configuration, e.g., surface minimization, and global geometric constraints [52, 67].

We review recent work that seeks to explain how cells migrate and sort by studying these interactions in a few examples: in order of increasing complexity: chick embryo cells, slime mold amoebae Dictyostelium dis- 


\begin{tabular}{|llll|ll|lllll|}
\hline 1 & 1 & 1 & 1 & 2 & 2 & 4 & 4 & 4 & 4 & 4 \\
1 & 1 & 1 & 2 & 2 & 2 & 2 & 4 & 4 & 4 & 4 \\
1 & 1 & 2 & 2 & 2 & 2 & 2 & 4 & 4 & 4 & 4 \\
1 & 1 & 2 & 2 & 2 & 2 & 4 & 4 & 4 & 4 & 4 \\
& 3 & 3 & 2 & 2 & 5 & 5 & 5 & 4 & 4 & 4 \\
3 & 3 & 3 & 3 & 3 & 5 & 5 & 5 & 5 & 4 & 4 \\
3 & 3 & 3 & 3 & 5 & 5 & 5 & 5 & 5 & 5 & 7 \\
3 & 3 & 3 & 3 & 5 & 5 & 5 & 7 & 7 & 7 & 7 \\
3 & 3 & 6 & 6 & 6 & 6 & 6 & 7 & 7 & 7 & 7 \\
3 & 3 & 6 & 6 & 6 & 6 & 6 & 6 & 7 & 7 & 7 \\
\hline
\end{tabular}

FIG. 8. Schematic of a two-dimensional cellular pattern represented in the large$Q$ Potts model. Numbers show different index values. Heavy black lines indicate cell boundaries [67].

coideum, and tumor growth. We introduce the Potts model in the context of grain growth where it was first developed as a cellular model, and extend it to describe morphogenesis.

3.1. Background of the $Q$-state Potts model. In the early 1980s, Anderson, Grest, Sahni and Srolovitz used the $Q$-state Potts model to study cellular pattern coarsening in metallic grains [130]. They treated the interior of a grain as containing "atoms" (each with a single index $\sigma$, describing the atom's crystalline orientation) distributed on a fixed lattice and the grain boundaries as the interfaces between different types of atoms or different crystal orientations. The total number of allowed states is $Q$. Figure 8 shows a schematic of a two-dimensional cellular pattern in the large- $Q$ Potts model.

The model starts from a free energy, the Potts Hamiltonian $\mathcal{H}$. In grain growth, the interface energy of domain boundaries is the only energy in the material, so the free energy is proportional to the boundary area of the domains, which is the number of mismatched links (i.e. neighboring lattice sites with different indices) [130]:

$$
\mathcal{H}=\sum_{\vec{i}, \vec{j}}\left[1-\delta_{\sigma(\vec{i}), \sigma(\vec{j})}\right]
$$

where $\sigma$ has $Q$ different values, typically integers from 1 to $Q ; J$ is the coupling energy between two unlike indices, thus corresponding to energy per unit area of the domain interface. The summation is over neighboring lattice sites $\vec{i}$ and $\vec{j}$. When the number of connected subdomains of different indices is comparable to $Q$ we say the model is "Large $Q . "$ If the number of connected subdomains is large compared to $Q$ then the model is "Small $Q$. ." 
Monte Carlo simulations of $Q$-state Potts models have traditionally employed local algorithms such as that of Metropolis et al. [98]. A lattice site is chosen at random and a new trial index is also chosen at random from one of the other $Q-1$ spins. The choice of the trial index is a somewhat delicate statistical mechanics problem (See [169]). The probability of changing the index at the chosen lattice site to the new index is:

$$
P= \begin{cases}1 & \Delta \mathcal{H} \leq 0 \\ \exp (-\Delta \mathcal{H} / T) & \Delta \mathcal{H}>0\end{cases}
$$

where $\Delta \mathcal{H}=\mathcal{H}_{\text {after }}-\mathcal{H}_{\text {before }}$ denotes the difference between the total energy before and after the index reassignment, and $T$ is the temperature. A Potts model simulation measures time in Monte Carlo Steps (MCS): one MCS is defined as as many trial substitutions as the number of lattice sites. Over time, these spin reassignments minimize the total domain interface energy.

Lattice simulations of surface energy run into difficulties when lattice discretization results in strong lattice anisotropy. In low temperature Potts model simulations, boundaries tend to align preferentially along low-energy orientations. In addition, boundaries can lock in position because the energy required to misalign a boundary in order to shorten it becomes too high. As a result, the pattern unrealistically traps in metastable higher energy states. Holm et al. [61] studied the effects of lattice anisotropy and temperature on coarsening in the large- $Q$ Potts model. Although by very different mechanisms, increasing temperature or using a longer-range interaction, e.g., fourth-nearest-neighbors on a square lattice, can both overcome the anisotropy inherent in discrete lattice simulations.

3.2. Extensions of the Potts model to biological applications. Over the last decade, extensions of the large- $Q$ Potts model have incorporated different aspects of biological cells [53, 60, 68, 93, 122].

In these applications, the domains of lattice sites with the same index describe cells, while links between lattice sites with different indices correspond to cell surfaces. The extensions fall into the following categories:

- Coupling between spins.

- Coupling to external fields.

- Constraints.

We review how to implement these extensions.

3.2.1. Coupling between spins. Cells adhere to each other using cell adhesion molecules (CAMs) present in the cell membrane [5]. Usually cells of the same type have the same CAMs and adhere to each other more strongly than to different types of cells (though certain CAMs adhere more strongly to molecules of different types). Glazier and Graner [53] incorporated this type-dependent adhesion into the Potts model by assigning "types" to indices, and assigning different coupling energies to different 
pairs of types. Smaller values of this energy correspond to stronger binding. To model an aggregate consisting of two randomly mixed cell types floating in a fluid medium, they simulated three types of cells: dark cells (d), light cells (l) and a fluid medium (M) that they treated as a generalized cell. The surface energy becomes [53]:

$$
\mathcal{H}_{1}=\sum_{\vec{i}, \vec{j}} J_{\tau(\sigma(\vec{i})), \tau(\sigma(\vec{j}))}\left(1-\delta_{\sigma(\vec{i}), \sigma(\vec{j})}\right)
$$

where $\tau(\sigma)$ is the type of cell $\sigma$. The summation is always over all neighboring sites in the lattice. We can transform the cell-type dependent coupling constants into surface tensions [53], and the total energy then corresponds to the appropriate surface tensions times the interface areas between the respective types.

A constant $J_{\tau, \tau^{\prime}}$ assumes that the cells are isotropic, which is only true for mesenchyme. Other tissues, such as epithelia, myocytes, or neurons, are polar, i.e. their cytoskeletons have established a direction, distinguishing top, bottom and side surfaces of the cells. An angular dependent coupling $J_{\tau, \tau^{\prime}}$, such as that in [168] can model cell polarity.

Cell-cell interactions are adhesive, thus the coupling energy is negative. While the change from positive to negative $J$ does not affect $\Delta H$, it does affect the hierarchy of energies with respect to the zero energy of an absent bond. Thus, simulations employing positive energies produce incorrect hierarchies of diffusion constants: more cohesive cells diffuse faster than less cohesive cells, contradicting common sense and experiments [149]. However, if we use a negative coupling strength, $J<0$, for the surface energy, the membrane breaks up to try to maximize its surface area (and hence minimize its energy). To recover the correct behavior we need to recognize that biological cells have a fixed amount of membrane which constrains their surface areas and at the same time reorganize to minimize their contact energy per unit surface. If we add an area constraint term resembling the volume constraint to the total energy and employ negative contact energies we recover the experimental diffusion behaviors [149].

3.2.2. Coupling to an external field. We can model directed cell motion, e.g., a cell's chemotactic motion where external chemical gradients guide cell movement in the direction of higher or lower chemical concentration, by coupling the index to an external field [68, 131]. The coupling pushes the cell boundaries, causing boundary migration and cell motion. The modification to the energy is:

$$
\mathcal{H}_{2}=\mathcal{H}_{1}+\sum_{\text {sites }} \mu C_{\vec{i}}
$$

where $\mu$ is the chemical potential, $C_{\vec{i}}$ is the chemical concentration at site $\vec{i}$, and the summation is over lattice sites experiencing chemotaxis. $\Delta \mathcal{H}^{\prime}=$ 
$\Delta \mathcal{H}+\mu\left(C_{\vec{i}^{\prime}}-C_{\vec{i}}\right)$. For a positive $\mu$, if $C>C^{\prime}$ then $\Delta \mathcal{H}^{\prime}<\Delta \mathcal{H}$ and the probability of accepting the reassignment increases. Over time, boundaries move more often into sites with higher concentrations, and the cell migrates up the chemical gradient. We can change the direction of chemotaxis by simply changing the sign of $\mu$. This simple choice for the chemical potential energy means that the cell velocity is proportional to the gradient of the chemical potential, i.e. the chemical concentration behaves like a potential energy. More complicated response function to chemical concentration are also possible.

3.2.3. Constraints. Biological cells generally have a fixed range of sizes (exceptions include the enucleate cells of the cornea and syncytal algae, myocytes, etc.). They do not grow or shrink greatly in response to their surface energy, though a small change in cell volume results from osmotic pressure. In the CPM, non-local forces such as those depending on cell volume or substrate curvature have the form of a Lagrangian constraint. Such a constraint term exacts an energy penalty for constraint violation. Glazier and Graner [53] described a cell volume constraint as an elastic term with cell rigidity $\Gamma$, and a fixed target size for the cell $V$. The total energy becomes:

$$
\mathcal{H}_{3}=\mathcal{H}_{1}+\sum_{\sigma} \Gamma(\tau(\sigma))[v(\sigma)-V(\tau(\sigma))]^{2},
$$

where $v(\sigma)$ is the volume of cell $\sigma$ and $V(\tau(\sigma))$ is the type-dependent target volume. Deviation from the target volume increases the total energy and therefore exacts a penalty. If we allow the target volumes to change in time, $V=V(t)$, we can model a variety of growth dynamics, such as cell growth as a function of nutrient supply (e.g., cancerous cell growth [117, 145]). Section 3.5 discusses the tumor growth model in more detail.

3.2.4. Extensions to Boltzmann evolution dynamics. The formation and breakage of CAM bonds is dissipative. Therefore we must modify the classical Boltzmann index evolution dynamics to include an explicit dissipation. Hogeweg et al. changed the probability for accepting index reassignments to reflect this dissipation [59]:

$$
P= \begin{cases}1 & \Delta \mathcal{H} \leq H_{\text {diss }} \\ \exp (-\Delta \mathcal{H} / T) & \Delta \mathcal{H}>H_{\text {diss }}\end{cases}
$$

where $H_{\text {diss }}$ represents the dissipation costs involved in deforming a boundary.

3.2.5. The complete cellular Potts model. With all these extensions, the CPM becomes a powerful cell level model for morphogenesis.

Savill et al. [131] and Jiang et al. [68] have independently developed CPMs that include differential adhesion and chemotaxis as the major intercellular interactions. The total energy is: 


$$
\mathcal{H}=\sum_{\vec{i}} \sum_{\vec{j}} \mathcal{J}_{\tau\left(\sigma_{\vec{i}}\right), \tau\left(\sigma_{\vec{j}}\right)}\left(1-\delta_{\sigma_{\vec{i}}, \sigma_{\vec{j}}}\right)+\Gamma \sum_{\sigma}\left[v_{\sigma}-V_{\sigma}\right]^{2}+\sum_{i} \mu C(i, t)
$$

The first term in the energy is the cell-type dependent adhesion energy. The second term encodes all bulk properties of the cell, such as membrane elasticity, cytoskeletal properties and osmotic pressure. The third term corresponds to chemotaxis, where the chemical potential determines if cells move towards or away from higher chemical concentrations. Varying the surface energies $J$ and the chemical potential $\mu$ tunes the relative strength between differential adhesion and chemotaxis [68].

3.3. Chicken cell sorting. The gist of Steinberg's Differential Adhesion Hypothesis (DAH) [142, 143] is that cells behave like immiscible fluids. Adhesive and cohesive interactions between cells generate surface and interfacial tensions. The analogy between cell sorting and the separation of immiscible fluids provides important quantitative information on the effective binding energy between cell adhesion molecules in situ under near-physiological conditions [11].

In chicken embryo cell-aggregate experiments a random mixture of two cell types sorts to form homotypic domains, as the upper panel in Figure 9 shows [102]. The simulations, with only differential adhesion and no chemotaxis, agree quantitatively with the experiment (Figure 9, lower panel) [102], validating the model.

In a liquid mixture, interfacial tension between the two phases, $\lambda$, drives hydrodynamic coalescence. When the volume fraction of the minority phase exceeds a "critical" value, its domains interconnect. The mean size of an interconnected domain, $L$, increases linearly in time [137]. Beysens et al. [11] found that in cellular aggregates, such as those shown in the top panels of Figure 9, the size of the interconnected domains also grows linearly in time [11], confirming the analogy between cells and immiscible fluids. Beysens et al. [11] also compared the coalescence dynamics of fluid mixtures to cell motion during sorting to define the membrane fluctuation energy in terms of the thermal energy $k_{B} T$. The numerical values of the membrane fluctuation energy translate into the binding energy between the adhesion molecules residing on the cell surfaces. Further experiments in quantifying these interactions will calibrate the cellular model and allow realistic choice of simulation temperatures.

3.4. Dictyostelium aggregation and culmination. One of the most widely used organisms in the study of morphogenesis is the slime mold Dictyostelium discoideum. It exhibits many general developmental processes including chemotaxis, complex behavior through self-organization, cell sorting and pattern formation. It has become a standard test for cellular models [68, 94, 131].

Unicellular amoebae, Dictyostelium, inhabit soil and eat bacteria. When starved, some pacemaker cells spontaneously emit pulses of the dif- 


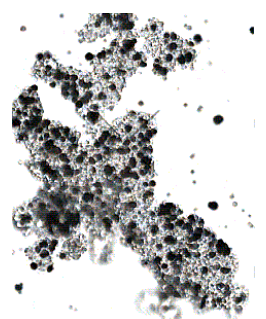

(a)

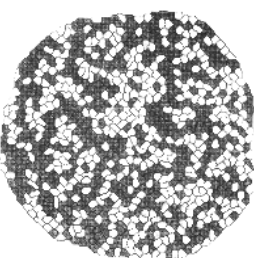

(a)

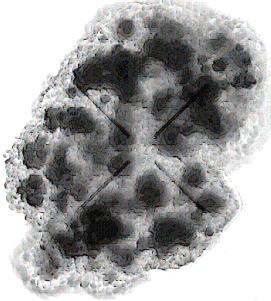

(b)

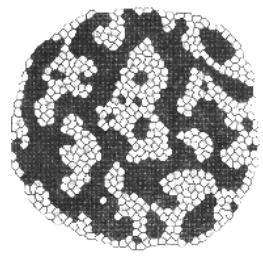

(b)

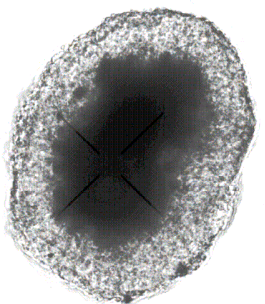

(c)

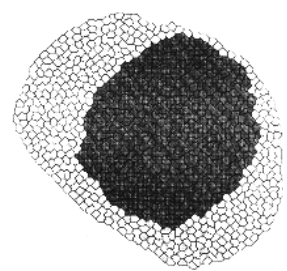

(c)

FIG. 9. Comparing the Cellular Potts Model simulation to a cell sorting experiment using chick retinal cells. The top panels show experimental images from chicken embryo cells in culture: light cells are neural retinal cells and dark cells are pigmented retinal cells. An initial random mixture of light and dark cells (a) forms dark clusters after around 10 hours (b), and eventually sorts to produce a dark cell core surrounded by light cells after around 72 hours. The bottom panels show the corresponding images from a simulation with three cells types: light cells, dark cells and medium [102].

fusing chemical signal cyclic adenosine monophosphate (cAMP), thereby initiating an excitation wave which propagates outward as a concentric ring or a spiral wave [17]. A neighboring cell responds to such a signal by elongating, moving a few micrometers up the gradient towards the source of cAMP, and synthesizing and releasing its own pulse of cAMP, attracting neighboring cells. This relaying results in cell-to-cell propagation of the cAMP signal [17]. Cells also release phosphodiesterase, which degrades cAMP to a null-signal, preventing the extracellular cAMP from building up to a level that swamps any gradients. The amoebae form streams when they touch each other and then form a multicellular mound, a hemispherical structure consisting of about $10^{5}-10^{6}$ cells, surrounded by a layer of slimy sheath. The cells in the mound then differentiate into two major types, pre-stalk (PST) cells (about 20\% of the cells) and pre-spore (PSP) cells (about 80\%) [88, 158]. Subsequently, the initially randomly distributed PST cells move to the top of the mound and form a protruding 


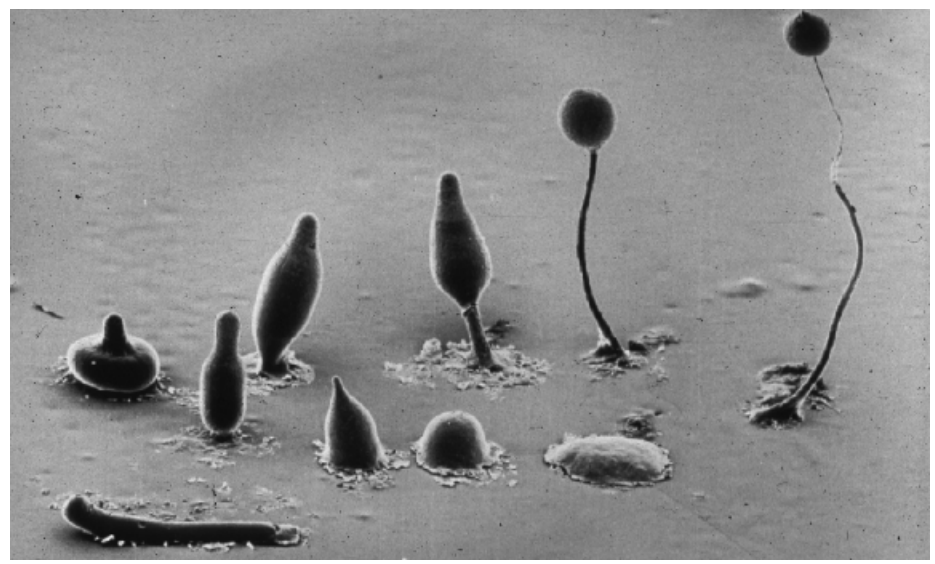

FIG. 10. Life cycle of Dictyostelium starting from a cell aggregate. The individual cells are about $10 \mu \mathrm{m}$ in diameter. The final fruiting body is about $3 \mathrm{~mm}$ tall. The whole cycle from starvation to culmination takes about 24 hours (courtesy of W. Loomis).

tip. This tip controls all morphogenetic movements during later multicellular development [127]. The elongated mound bends over and migrates as a multicellular slug. When the slug stops, the tip (the anterior part of the slug) sits on a somewhat flattened mound consisting of PSP cells. The tip then retracts and the stalk (formerly PST) cells elongate and vacuolate, pushing down through the mass of spore (formerly PSP) cells. This motion hoists the mass of spore cells up along the stalk. The mature fruiting body consists of a sphere of spore cells sitting atop a slender tapering stalk. The whole life cycle, from starvation to formation of the fruiting body, shown in Figure 10, normally takes about 24 hours.

Various stages of the Dictyostelium life cycle have been modeled using continuum approaches. Classical two-dimensional models for aggregation date back to early 1970 s $[75,106]$. Othmer et al. recently proposed "Chemotaxis equations" as the diffusion approximation of transport equations [113], which use external biases imposed on cell motion to modify cell velocity or turning rate and describe chemotaxis aggregation phenomenologically for both myxobacteria and Dictyostelium. Odell and Bonner modeled slug movement [110] using a mechanical description where cells respond to cAMP chemotactically and the active component of the propulsive force enters as a contribution to the stress tensor. Vasiev et al. [152] also included cAMP dynamics in a continuum model of Dictyostelium cell movement. Their model adds forces corresponding to chemotaxis to the Navier-Stokes equations. Although they can produce solutions that resemble aggregation, their equations do not include an elastic response, making it difficult to connect the forces postulated with experimentally measurable quantities. 
As chemotaxis is an important aspect of Dictyostelium development, the cellular model requires an additional field to describe the local concentration $C$ of cAMP diffusing in extracellular space. The equation for the field is:

$$
\frac{\partial C(\vec{x})}{\partial t}=D \nabla^{2} C-\gamma C+S_{c}(S, \vec{x}, t) .
$$

where $D$ is the diffusion constant of cAMP; $\gamma$ is its decay rate; the source term $S_{c}$ describes cAMP being secreted or absorbed at the surface of cells, whose specific form requires experimental measurement of the cAMP concentrations in the tissue.

Using the cellular model coupled to the reaction-diffusion equation for a general chemo-attractant, Marée et al. [93] were able to simulate the entire life-cycle of Dictyostelium. Features they have added to the cellular model include:

- treating chemotaxis as periodic cell movement during aggregation, slug migration, and culmination,

- describing cAMP dynamics inside the cells by an ODE, the two variable FitzHugh-Nagumo equation [92],

- assuming that contact between the cell types determines cell differentiation and modeling an irreversible conversion of cell types during culmination: PstO cells differentiate into PstA cells, and PstA cells into stalk cells,

- biasing the index transition probability $p$, with a high $H_{\text {diss }}$ to represent the stiffness of the stalk tube.

They also assumed that a special group of pathfinder cells occupies the tip region of the elongating stalk, guiding the stalk downwards. Figure 11 shows the full cycle of culmination from a mound of cells into a fruiting body.

Hogeweg et al. [59] further extended the cellular model to allow cells internal degrees of freedom to represent genetic information, which then controls cell differentiation under the influence of cell shape and contacts. Open questions include how cells polarize in response to the chemotactic signal, how they translate this information into directed motion, how cells move in a multicellular tissue, and the role of differential cell adhesion during chemotactic cell sorting. We may be able to answer these questions using the CPM since we can control the relative importance of differential adhesion and chemotaxis (e.g., as in [68]) and include cell polarity models (e.g., as in [168]).

A two-dimensional experiment on Dictyostelium aggregation (by trapping the cells between agar plates) by Levine et al. [85] found that the cells organize into pancake-like vortices. Rappel et al. used a two-dimensional extension of the CPM to model such aggregation [122]: aggregation and vortex motion occur without a diffusing chemoattractant provided the initial cell density is sufficiently high. In addition to the generic CPM with cell 


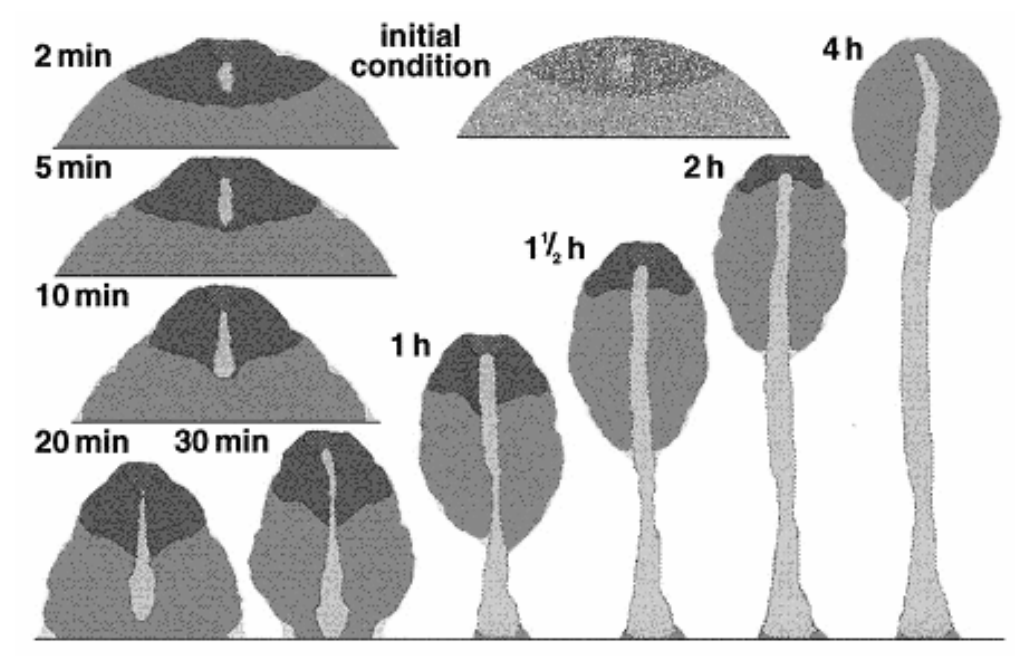

FIG. 11. Simulation of the culmination of Dictyostelium using the CPM coupled to reaction-diffusion dynamics for diffusing cAMP. Gray scales encode different cell types. Over time, the stalk cells push down through the mass of spore cells and hoist the sphere of spore cells up along the stalk [94] (courtesy of S. Marée).

adhesion and a volume constraint, their model includes a cell-generated motive force to model the cell's cytoskeleton-generated front protrusions and back retractions, using a local potential energy. They also assume that each cell changes the direction of its cytoskeletal force to match those of neighboring cells. With these assumptions, cells self-organize into a roughly circular, rotating, confluent vortex. The model reproduces the experimental observations that confluent cells move faster than isolated cells and that cells slip past each other in a rotating aggregate. The angular velocity of cells as a function of radial location in the aggregate agrees with experiment ([122]). The implication of this paper, however, is not clear. The simulation seems to suggest that the vortex arises from local cell interactions without chemotaxis, as seen in many swarm models, whereas most researchers believe that chemotaxis is present during aggregation and is responsible for the collective motion of Dictyostelium.

3.5. Tumor growth. Another example that illustrates the capabilities of the CPM is modeling tumor growth. Exposure to ultra-violet radiation, toxic chemicals, and byproducts of normal metabolism can all cause genetic damage [76]. Some abnormal cells grow at a rate exceeding the growth rate of normal surrounding tissue and do not respond to signals to stop cell division [5]. During cell division, these changes can accumulate and multiply. In some cases cells can become cancerous. The cancer becomes malignant if the cells detach from the parent tumor (metastasize) 
and migrate to a distant location and form secondary tumors. Thus cancers involve both a failure of cell differentiation and of cell migration [76].

Even though the basic processes of tumor growth are understood, predicting the evolution of a tumor in vivo is beyond current numerical tools. A large number of factors influence tumor growth, e.g., the type of the cancerous cells, local nutrient and waste concentrations, the anatomy and location of the tumor, etc. The secretion by the tumor of endothelial growth factors which induce the growth of new blood vessels which supply nutrients to the tumor (angiogenesis) is particularly complex. Even in in vitro experiments with well controlled microenvironments, stochastic effects that are always present make prediction difficult. The first step of tumor growth, an avascular tumor that grows into a spherical, layered structure consisting of necrotic, quiescent and proliferating cells, is more tractable. Multicellular tumor spheroid (MTS) experiments as an in vitro tumor model can provide data on the duration of the cell cycle, growth rate, chemical diffusion, etc. $[48,49]$.

Tumor growth requires the transport of nutrients (e.g., oxygen and glucose) from and waste products to the surrounding tissue. These chemicals regulate cell mitosis, cell death, and potentially cell mutation. MTS experiments have the great advantage of precisely controlling the external environment while maintaining the cells in the spheroid microenvironment $[48,49]$. Suspended in culture, tumor cells grow into a spheroid, in a process that closely mimics the growth characteristics of early stage tumors. MTS exhibit three distinct phases of growth: 1) an initial phase during which individual cells form small clumps that subsequently grow quasiexponentially; 2) a layering phase during which the cell-cycle distribution within the spheroid changes, leading to formation of a necrotic core, accumulation of quiescent cells around the core, and sequestering of proliferating cells at the periphery; and 3) a plateau phase during which the growth rate begins to decrease and the tumor ultimately attains a maximum diameter. Freyer et al. $[48,49]$ use EMT6/ $R_{0}$ mouse mammary tumor spheroids and provide high-precision measurements for controlled glucose and oxygen supply, as well as various inhibition factors and growth factors. Abundant data are also available in the literature on the kinetics of tumor growth under radiation treatment or genetic alteration [76].

Numerous models have analyzed the evolution of cell clusters as a simplified tumor [1]. Approaches include:

1. Continuum models including those using classical growth models such as the von Bertalanffy, logistic or Gompertz models [95, 96]. Among them, the Gompertz model best fits experimental data. None of these rate models (empirical ordinary differential equations) can simulate the evolution of tumor structure, or predict the effect of chemicals on tumor morphology.

2. CA models that treat cells as single points on a lattice, e.g., the LGCA model of Dormann and Deutsch [36]. They adopt local rules speci- 
fying adhesion, pressure (cells are pushed towards regions of low cell density) and couple the LGCA to a continuum chemical dynamics. Their two-dimensional simulations produce a layered structure that resembles a cross-section of an MTS.

3. Biomechanical models using finite-element methods (e.g., [81]), mostly applied to brain and bone tumors. These models emphasize the soft-tissue deformations induced by tumor growth.

We now describe how the CPM can model tumor growth. Any model of tumor growth must consider cell-cell adhesion, chemotaxis, cell dynamics including cell growth, cell division and cell mutation, as well as the reaction-diffusion of chemicals: nutrients and waste products, and eventually, angiogenesis factors and hormones. In additional to differential adhesion and chemotaxis, Jiang et al. [117] include in their cellular model the reaction-diffusion dynamics for relevant chemicals:

$$
\begin{aligned}
\frac{\partial C_{o}}{\partial t} & =D_{o} \nabla^{2} C_{o}-a(\vec{x}), \\
\frac{\partial C_{n}}{\partial t} & =D_{n} \nabla^{2} C_{n}-b(\vec{x}), \\
\frac{\partial C_{w}}{\partial t} & =D_{w} \nabla^{2} C_{w}+c(\vec{x}) .
\end{aligned}
$$

where $C_{o}, C_{n}$ and $C_{w}$ are the concentrations of oxygen, nutrients (e.g., glucose) and metabolic wastes (e.g., lactate), $d_{o}, d_{n}$ and $d_{w}$ are their respective diffusion constants; $a$ and $b$ are the metabolic rates of the cell located at $\vec{x}$; and $c$ is the coefficient of metabolic waste production, which depends on $a$ and $b$. Each cell follows its own cell cycle, which depends sensitively on its local chemical environment. The target volumes are twice the initial volumes. The volume constraint in the total energy allows cell volumes to stay close to the target volume, thus describing cell growth. If the nutrient concentration falls below a threshold or the waste concentration exceeds its threshold, the cell stops growing and become quiescent: alive but not growing. When the nutrient concentration drops lower or waste increases further, the quiescent cell may become necrotic. Only when the cell reaches the end of its cell cycle and its volume reaches a target volume will the cell divide. The mature cell then splits along its longest axis into two daughter cells, which may inherit all the properties of the mother cell or undergo mutation with a defined probability.

The simulation data show that the early exponential stage of tumor growth slows down when quiescent cells appear [117]. Other measurements also qualitatively reproduce experimental data from multicellular spheroids grown in vitro. These simulations model a monoclonal cell population in accordance with MTS experiments. However, including cellular heterogeneity as e.g., in the model of Kansal et al. [73] is straightforward. Model extensions will incorporate genetic and epigenetic cell heterogeneity. The 
CPM allows easy implementation of cell differentiation as well as additional signal molecules.

4. Summary. Physical parameters such as energy, temperature and compressibility combined with processes such as energy minimization and reaction-diffusion of chemicals control the evolution and properties of both living and nonliving materials. We can describe surprisingly complex living organisms simply by combining these classical physical concepts. Why are living structures often so elaborate? The complexity arises in two ways: first as an emergent property of the interaction of a large number of autonomously motile cells that can self-organize. Cells need not form thermodynamically equilibrated structures. Second, cells have a complex feedback interaction with their environment. Cells can modify their surroundings by e.g., secreting diffusible or non-diffusible chemicals. Their environment in turn causes changes in cell properties (differentiation) by changing the levels of gene expression within the cell.

Cellular automaton models describe cell-cell and cell-environment interactions by phenomenological local rules, allowing simulation of a huge range of biological examples ranging from bacteria and slime model amoebae, to chicken embryonic tissues and tumors.

\section{REFERENCES}

[1] J. Adam and N. Bellomo, A survey of models for tumor-immune system dynamics, Birkhauser, Boston, 1997.

[2] A. Adamatzky and O. Holland, Phenomenology of excitation in 2-D cellular automata and swarm systems, Chaos Solitons \& Fractals, 9 (1998), pp. 12331265 .

[3] M. Alber and M. Kiskowski, On aggregation in CA models in biology, J. Phys. A: Math. Gen., 34 (2001), pp. 10707-10714.

[4] M. Alber, M. Kiskowski, AND Y. JiAng, A model of rippling and aggregation in Myxobacteria, 2002 preprint.

[5] B. Alberts, M. Raff, J. Watson, K. Roberts, D. Bray, and J. Lewis, Molecular biology of the cell, 3rd edition, Garland Publishing, NY, 1994.

[6] J. Ashkin And E. Teller, Statistics of two-dimensional lattices with four components, Phys. Rev., 64 (1943), pp. 178-184.

[7] E. Ben-Jacob, I. Cohen, A. Czirk, T. Vicsek, and D.L. Gutnick, Chemomodulation of cellular movement, collective formation of vortices by swarming bacteria, and colonial development, Physica A, 238 (1997), pp. 181-197.

[8] E. Ben-Jacob and H. Levine, The artistry of microorganisms, Scientific American, 279 (1998), pp. 82-87.

[9] E. Ben-Jacob, I. Cohen, and H. Levine, Cooperative self-organization of microorganisms, Advances in Physics, 49 (2000), pp. 395-554.

[10] L. Besseau and M. Giraud-Guille, Stabilization of fluid cholesteric phases of collagen to ordered gelated matrices, J. Mol. Bio., 251 (1995), pp. 137-145.

[11] D. Beysens, G. Forgacs, and J.A. Glazier, Cell sorting is analogous to phase ordering in fluids, Proc. Natl. Acad. Sci. USA 97 (2000) pp. 9467-9471.

[12] H. Bode, K. Flick, and G. Smith, Regulation of interstitial cell-differentiation in Hydra attenuata. I. Homeostatic control of interstitial cell-population size, J. Cell Sci., 20 (1976), pp. 29-46. 
[13] E. Bonabeau, M. Dorigo, and G. Théraulaz, Swarm intelligence: From natural to artificial systems, Oxford University Press, NY, 1999.

[14] J. Boon., D. DAB, R. KApral, And A. LawniczAK, Lattice gas automata for relative systems, Physics Reports, 273 (1996), pp. 55-147.

[15] U. Börner, A. Deutsch, H. Reichenbach, and M. Bar, Rippling patterns in aggregates of myxobacteria arise from cell-cell collisions, 2002 preprint.

[16] H. Bussemaker, A. Deutsch, and E. Geigant, Mean-field analysis of a dynamical phase transition in a cellular automaton model for collective motion, Phys. Rev. Lett., 78 (1997), pp. 5018-5027.

[17] M. Caterina And P. Devreotes, Molecular insights into eukaryotic chemotaxis, FASEB J., 5 (1991), pp. 3078-3085.

[18] S. Chen, S.P. Dawson, G.D. Doolen, D.R. Janecky, and A. Lawniczak, Lattice methods and their applications to reacting systems, Computers \& Chemical Engineering, 19 (1995), pp. 617-646.

[19] B. Chopard and M. Droz, Cellular automata modeling of physical systems, Cambridge University Press, NY, 1998.

[20] I. Cohen, I.G. Ron, And E. Ben-JacoB, From branching to nebula patterning during colonial development of the Paenibacillus alvei bacteria, Physica A, 286 (2000), pp. 321-336.

[21] J. Cook, Waves of alignment in populations of interacting, oriented individuals, Forma, 10 (1995), pp. 171-203.

[22] J. Cook, A. Deutsch, AND A. Mogilner, Models for spatio-angular selforganization in cell biology, in W. Alt, A. Deutsch and G. Dunn (Eds.) Dynamics of cell and tissue motion, Birkhuser, Basel, Switzerland, 1997, pp. 173-182.

[23] M. Cross and P. Hohenberg, Pattern-formation outside of equilibrium, Rev. Mod. Phys., 65 (1993), pp. 851-1112.

[24] A. Czirok, A. L. Barabasi, and T. Vicsek, Collective motion of organisms in three dimensions, Phys. Rev. Lett., 82 (1999), pp. 209-212.

[25] J. Dallon and J. Sherratt, A mathematical model for spatially varying extracellular matrix alignment, SIAM J. Appl. Math., 61 (2000), pp. 506-527.

[26] L.A. Davidson, M.A.R. Koehl, R. Keller, and G.F. Oster, How do sea-urchins invaginate - Using biomechanics to distinguish between mechanisms of primary invagination, Development, 121 (1995), pp. 2005-2018.

[27] A.M. Delprato, A. Samadani, A. Kudrolli, and L.S. Tsimring, Swarming ring patterns in bacterial colonies exposed to ultraviolet radiation, Phys. Rev. Lett., 87 (2001), 158102.

[28] A. Deutsch, Towards analyzing complex swarming patterns in biological systems with the help of lattice-gas automaton model, J. Biol. Syst., 3 (1995), pp. 947-955.

[29] A. Deutsch, Orientation-induced pattern formation: Swarm dynamics in a latticegas automaton model, Int. J. Bifurc. Chaos, 6 (1996), pp. 1735-1752.

[30] A. Deutsch, Principles of morphogenetic motion: swarming and aggregation viewed as self-organization phenomena, J. Biosc., 24 (1999), pp. 115-120.

[31] A. Deutsch, Probabilistic lattice models of collective motion and aggregation: from individual to collective dynamics, Mathematical Biosciences, 156 (1999), pp. 255-269.

[32] A. Deutsch, A new mechanism of aggregation in a lattice-gas cellular automaton model, Mathematical and Computer Modeling, 31 (2000), pp. 35-40.

[33] A. Deutsch and S. Dormann, Cellular Automata and Biological Pattern Formation Modeling, 2002 preprint.

[34] S. Dormann, Pattern Formation in Cellular Automation Models, Dissertation, Angewandte Systemwissenschaft FB Mathematik/Informatik, Universität Osnabrück, Austria, 2000.

[35] S. Dormann, A. Deutsch, and A. Lawniczak, Fourier analysis of Turing-like pattern formation in cellular automaton models, Future Computer Generation Systems, 17 (2001), pp. 901-909. 
[36] S. Dormann And A. Deutsch, Modeling of self-organized avascular tumor growth with a hybrid cellular automaton, Silico Biology, 2 (2002), 0035.

[37] D. Drasdo and G. Forgacs, Modeling the interplay of generic and genetic mechanisms in cleavage, blastulation, and gastrulation, Developmental Dynamics, 219 (2000), pp. 182-191.

[38] M. Dworkin and D. Kaiser, Myxobacteria II, American Society for Microbiology, Washington, DC, 1993.

[39] M. Dworkin Recent advances in the social and developmental biology of the myxobacteria, Microbiol. Rev., 60 (1996), pp. 70-102.

[40] M. Eden, Vol. 4: Contributions to biology and problems of medicine, in J. Neyman (Ed.), Proceedings of the Fourth Berkeley Symposium in Mathematics, Statistics and Probability, University of California Press, Berkeley, 1961, pp. 223-239.

[41] R. Engelhardt, Modeling pattern formation in reaction diffusion systems, Master's Thesis, Dept. of Chemistry, University of Copenhagen, Denmark, 1994.

[42] G. Ermentrout and L. Edelstein-Keshet, Cellular automata approach in biological modeling, J. Theor. Biol., 160 (1993), pp. 97-133.

[43] S.E. Esipov and J.A. Shapiro, Kinetic model of Proteus mirabilis swarm colony development J. Math. Biol., 36 (1998), pp. 249-268.

[44] M. Fontes AND D. KAISER, Myxococcus cells respond to elastic forces in their substrate, Proc. Natl. Acad. Sci. USA, 96 (1999), pp. 8052-8057.

[45] G. Forgacs, R. Foty, Y. Shafrir, and M. Steinberg, Viscoelastic properties of living embryonic tissues: a quantitative study, Biophys. J., 74 (1998), pp. 2227-2234.

[46] R. Foty, G. Forgacs, C. Pfleger, and M. Steinberg, Liquid properties of embryonic tissues: measurements of interfacial tensions, Phys. Rev. Lett., $\mathbf{7 2}$ (1994), pp. 2298-2300.

[47] R. Foty, C. Pfleger, G. Forgacs, and M. Steinberg, Surface tensions of embryonic tissues predict their mutual envelopment behavior, Development, 122 (1996), pp. 1611-1620.

[48] J. Freyer and R. Sutherland, Selective dissociation and characterization of cells from different regions of multicell spheroids during growth, Cancer Research, 40 (1980), pp. 3956-3965.

[49] J. Freyer AND R. Sutherland, Regulation of growth saturation and development of necrosis in EMT6/RO multicellular spheroids induced by the glucose and oxygen supply, Cancer Research, 46 (1986), pp. 3504-3512.

[50] M. Gardner, The fantastic combinations of John Conway's new solitaire game 'life', Scientific American, 223 (1970), pp. 120-123.

[51] F. Gianocotti, Integrin-signaling: specificity and control of cell survival and cell cycle progression, Curr. Opin. Cell Biol., 9 (1997), pp. 691-700.

[52] J.A. Glazier, Dynamics of cellular patterns, Ph.D. Thesis, The University of Chicago, USA, 1989.

[53] J.A. Glazier and F. Graner, Simulation of the differential adhesion driven rearrangement of biological cells, Phys. Rev. E, 47 (1993), pp. 2128-2154.

[54] D. Godt and U. Tepass, Drosophila oocyte localization is mediated by differential cadherin-based adhesion, Nature, 395 (1998), pp. 387-391.

[55] I. Golding, Y. Kozlovsky, I. Cohen, and E. Ben-Jacob, Studies of bacterial branching growth using reaction-diffusion models for colonial development, Physica A, 260 (1998), pp. 510-554.

[56] A. González-Reyes and D. St. Johnston, Patterning of the follicle cell epithelium along the anterior-posterior axis during Drosophila oogenesis, Development, 125 (1998), pp. 2837-2846.

[57] F. Graner and J.A. Glazier, Simulation of biological cell sorting using a twodimensional Extended Potts Model, Phys. Rev. Lett., 69 (1992), pp. 20132016 . 
[58] J. Hardy, O. De Pazzis, and Y. Pomeau, Molecular dynamics of a classical lattice gas: Transport properties and time correlation functions, Phys. Rev. A, 13 (1976), pp. 1949-1961.

[59] P. HogeweG, Evolving mechanisms of morphogenesis: On the interplay between differential adhesion and cell differentiation, J. Theor. Biol., 203 (2000), pp. 317-333.

[60] P. Hogeweg, Shapes in the shadow: Evolutionary dynamics of morphogenesis, Artificial Life, 6 (2000), pp. 611-648.

[61] E. Holm, J.A. Glazier, D. Srolovitz, and G. Grest, Effects of lattice anisotropy and temperature on domain growth in the 2-dimensional Potts model, Phys. Rev. A, 43 (1991), pp. 2262-2268.

[62] A. Howe, A. Aplin, S. Alahari, And R. Juliano, Integrin signaling and cell growth control, Curr. Opin. Cell Biol., 10 (1998), pp. 220-231.

[63] O. Igoshin, A. Mogilner, D. Kaiser, And G. Oster, Pattern formation and traveling waves in myxobacteria: Theory and modeling, Proc. Natl. Acad. Sci. USA, 98 (2001), pp. 14913-14918.

[64] L. Jelsbak and L. SøgaArd-Andersen, The cell surface-associated intercellular C-signal induces behavioral changes in individual Myxococcus xanthus cells during fruiting body morphogenesis, Devel. Bio, 96 (1998), pp. 5031-5036.

[65] L. Jelsbak and L. SøgaArd-Andersen, Pattern formation: Fruiting body morphogenesis in Myxococcus xanthus, Current Opinion in Microbiology, 3 (2000), pp. 637-642.

[66] Y. Jiang and J.A. Glazier, Extended large- $Q$ Potts model simulation of foam drainage, Philos. Mag. Lett., 74 (1996), pp. 119-128.

[67] Y. Jiang, Cellular pattern formation, Ph.D. Thesis, University of Notre Dame, USA, 1998.

[68] Y. Jiang, H. Levine, and J.A. Glazier, Possible cooperation of differential adhesion and chemotaxis in mound formation of Dictyostelium, Biophys. J., 75 (1998), pp. 2615-2625.

[69] B. Julien, D. Kaiser, And A. Garza, Spatial control of cell differentiation in Myxococcus xanthus, Proc. Natl. Acad. Sci. USA, 97 (2000), pp. 9098-9103.

[70] L.P. Kadanoff, G.R. McNamara, and G. Zanetti, From automata to fluidflow-Comparisons of simulation and theory, Phys. Rev. A, 40 (1989), pp. $4527-4541$.

[71] D. Kaiser, How and why myxobacteria talk to each other, Current Opinion in Microbiology, 1 (1998), pp. 663-668.

[72] D. KAISER, Intercellular Signaling For Multicellular Morphogenesis, Society for General Microbiology Symposium 57, Cambridge University Press, Society for General Microbiology Ltd., UK, 1999.

73] A. Kansal, S. Torquato, E. Chiocca, and T. Deisboeck, Emergence of a subpopulation in a computational model of tumor growth, J. Theor. Biol., 207 (2000), pp. 431-441.

[74] N. Kataoka, K. Saito, And Y. SaWAda, NMR microimaging of the cell sorting process, Phys. Rev. Lett., 82 (1999), pp. 1075-1078.

[75] E.F. Keller AND L.A. SEgal, Initiation of slime mold aggregation viewed as an instability, J. Theor. Bio., 26 (1970), pp. 399-415.

[76] P. Kiberstis and J. Marx, Frontiers in cancer research, Science, 278 (1977), pp. $1035-1035$.

[77] S. Kim AND D. Kaiser, Cell alignment in differentiation of Myxococcus xanthus, Science, 249 (1990), pp. 926-928.

[78] S. KIM AND D. KAISER, C-factor has distinct aggregation and sporulation thresholds during Myxococcus development, J. Bacteriol., 173 (1991), pp. 17221728 .

[79] M. Kiskowski, M. Alber, G. Thomas, J. Glazier, N. Bronstein, and S. NewMAN, Interaction between reaction-diffusion process and cell-matrix adhesion in a cellular automata model for chondrogenic pattern formation: a prototype study for developmental modeling, 2002, in preparation. 
[80] J. Kuner And D. KAISER, Fruiting body morphogenesis in submerged cultures of Myxococcus xanthus, J. Bacteriol., 151 (1982), pp. 458-461.

[81] S. Kyriacou, C. Davatzikos, S. Zinreich, and R. Bryan, Nonlinear elastic registration of brain images with tumor pathology using a biomechanical model, IEEE Transactions On Medical Imaging, 18 (1999), pp. 580-592.

[82] J. Landry, J. Freyer, and R. Sutherland, A model for the growth of multicellular spheroids, Cell Tiss. Kinet., 15 (1982), pp. 585-594.

[83] C. Leonard, H. Fuld, D. Frenz, S. Downie, Massagué, and S. Newman, Role of Transforming Growth Factor- $\beta$ in chondrogenic pattern formation in the embryonic limb: Stimulation of mesenchymal condensation and fibronectin gene expression by exogenous TGF- $\beta$-like activity, Devel. Bio., 145 (1991), pp. 99-109.

[84] H. Levine, I. Aranson, L. Tsimring, and T. Truong, Positive genetic feedback governs cAMP spiral wave formation in Dictyostelium, Proc. Natl. Acad. Sci. USA, 93 (1996), pp. 6382-6386.

[85] A. Nicol, W.J. Rappel, H. Levine, and W.F. Loomis, Cell-sorting in aggregates of Dictyostelium discoideum, J. Cell. Sci., 112 (1999), pp. 3923-3929.

[86] H. Levine, W-J. Rappel, And I. Cohen, Self-organization in systems of selfpropelled particles, Phys. Rev. E, 63 (2001), 017101.

[87] S. Li, B. Lee And L. Shimkets, csgA expression entrains Myxococcus Xanthus development, Genes Development, 6 (1992), pp. 401-410.

[88] W. Loomis, Lateral inhibition and pattern formation in Dictyostelium, Curr. Top. Dev. Biol., 28 (1995), pp. 1-46.

[89] F. Lutscher, Modeling alignment and movement of animals and cells, J. Math. Biol., DOI: 10.1007/s002850200146, 2002.

[90] F. Lutscher And A. Stevens, Emerging patterns in a hyperbolic model for locally interacting cell systems, Journal of Nonlinear Sciences, 2002 preprint.

[91] P. MAini, Mathematical models in morphogenesis, pp. 151-189. In V. Capasso and O. Dieckmann (Eds.), Mathematics Inspired Biology, Springer, Berlin, 1999.

[92] A. Marée, A. Panfilov, and P. Hogeweg, Migration and thermotaxis of Dictyostelium discoideum slugs, a model study, J. Theor. Biol., 199 (1999), pp. 297-309.

[93] A. MARÉE, From pattern formation to morphogenesis: Multicellular coordination in Dictyostelium discoideum, Ph.D. Thesis., Utrecht University, the Netherlands, 2000.

[94] A. Marée And P. Hogeweg, How amoeboids self-organize into a fruiting body: Multicellular coordination in Dictyostelium discoideum, Proc. Natl. Acad. Sci. USA, 98 (2001), pp. 3879-3883.

[95] M. Marusic, Z. Bajzer, J. Freyer, and S. Vuk-Pavlovic, Modeling autostimulation of growth in multicellular tumor spheroids, Int. J. Biomed. Comput., 29 (1991), pp. 149-158.

[96] M. Marusic, Z. Bajzer, J. Freyer, and S. Vuk-Pavlovic, Analysis of growth of multicellular tumor spheroids by mathematical models, Cell Prolif., 27 (1994), pp. $73-94$.

[97] J. Marrs and W. Nelson, Cadherin cell adhesion molecules in differentiation and embryogenesis, Int. Rev. Cytol., 165 (1996), pp. 159-205.

[98] N. Metropolis, A.W. Rosenbluth, M.N. Rosenbluth, A.H. Teller, and E. Teller, Combinatorial minimization, J. Chem. Phys., 21 (1953), pp. 10871092 .

[99] A. Mogilner and L. Edelstein-Keshet, Spatio-angular order in populations of self-aligning objects: formation of oriented patches, Physica D, 89 (1996), pp. 346-367.

[100] A. Mogilner, L. Edelstein-Keshet, And G. Ermentrout, Selecting a common direction. II. Peak-like solutions representing total alignment of cell clusters, J. Math. Biol., 34 (1996), pp. 811-842. 
[101] A. Mogilner and L. Edelstein-Keshet, A non-local model for a swarm, J. Math. Biol., 38 (1999), pp. 534-570.

[102] J. Mombach, J.A. Glazier, R. Raphael, and M. Zajac, Quantitative comparison between differential adhesion models and cell sorting in the presence and absence of fluctuations, Phys. Rev. Lett., 75 (1995), pp. 2244-2247.

[103] J. Mombach And J.A. Glazier, Single cell motion in aggregates of embryonic cells, Phys. Rev. Lett., 76 (1996), pp. 3032-3035.

[104] F. Monier-Gavelle And J. Duband, Cross talk between adhesion molecules: Control of $\mathrm{N}$-cadherin activity by intracellular signals elicited by beta 1 and beta 3 integrins in migrating neural crest cells, J. Cell. Biol., 137 (1997), pp. $1663-1681$.

[105] J. Murray, Mathematical Biology, Biomathematics 19, Springer, New York, 1989.

[106] V. NAnjundiah, Chemotaxis, signal relaying and aggregation morphology, J. Theor. Bio., 42 (1973), pp. 63-105.

[107] S. Newman and H. Frisch, Dynamics of skeletal pattern formation in developing chick limb, Science, 205 (1979), pp. 662-668.

[108] S. Newman, Sticky fingers: Hox genes and cell adhesion in vertebrate development, Bioessays, 18 (1996), pp. 171-174.

[109] K. O'Connor And D. Zusman, Patterns of cellular interactions during fruitingbody formation in Myxococcus xanthus, J. Bacteriol., 171 (1989), pp. 60136024 .

[110] G.M. Odell And J.T. Bonner, How the Dictyostelium discoideum grex crawls, Philos. Trans. Roy. Soc. London, B., 312 (1985), pp. 487-525.

[111] C. Ofria, C. Adami, T.C. Collier, and G.K. Hsu, Evolution of differentiated expression patterns in digital organisms; Lect. Notes Artif. Intell., 1674 (1999), pp. 129-138.

[112] H.G. Othmer, S. Dunbar, and W. Alt, Models of dispersal in biological systems, J. Math. Biol., 26 (1988), pp. 263-298.

[113] H.G. Othmer and T. Hillen, The diffusion limit of transport equations II: Chemotaxis equations, SIAM J. Appl. Math., 62 (2002), pp. 1222-1250.

[114] J.K. Parrish And W. Hamner, (Eds.), Animal groups in three dimensions, Cambridge University Press, Cambridge, 1997.

[115] J.K. Parrish and L. Edelstein-Keshet, From individuals to aggregations: Complexity, epiphenomena, and evolutionary trade-offs of animal aggregation, Science, 284 (1999), pp. 99-101.

[116] A. Pelizzola, Low-temperature phase of the three-state antiferromagnetic Potts model on the simple-cubic lattice, Phys. Rev. E, 54 (1996), pp. R5885-R5888.

[117] J. PJESIVAC AND Y. JIANG, A cellular model for avascular tumor growth, unpublished (2002).

[118] T. Pollard AND J. Cooper, Actin and actin-binding proteins. A critical evaluation of mechanisms and function, Ann. Rev. Biochem., 55 (1986), pp. 9871035.

[119] R. Potts, Some generalized order-disorder transformations, Proc. Cambridge Phil. Soc., 48 (1952), pp. 106-109.

[120] I. Prigogine And R. Herman, Kinetic theory of vehicular traffic, American Elsevier, New York, 1971.

[121] S. Rahman, E. Rush, and R. Swendsen, Intermediate-temperature ordering in a three-state antiferromagnetic Potts model, Phys. Rev. B. 58 (1998). pp. 91259130.

[122] W.J. Rappel, A. Nicol, A. Sarkissian, H. Levine, and W.F. Loomis Selforganized vortex state in two-dimensional Dictyostelium dynamics, Phys. Rev. Lett., 83 (1999), pp. 1247-1250.

[123] H. Reichenbach, Myxobacteria: A most peculiar group of social prokaryotes, in Myxobacteria development and cell interactions, E. Rosenburg (Ed.) SpringerVerlag, NY, 1984, pp. 1-50.

[124] C.W. ReYnolds, Flocks, herds, and schools: A distributed behavioral model, ACM Computer Graphics, SIGGRAPH '87, 21 (1987), pp. 25-34. 
[125] D. Richardson Random growth in a tessellation, Proc. Camb. Phil. Soc., 74 (1973), pp. 563-573.

[126] J. Rieu, A. Upadhyaya, J.A. Glazier, N. Ouchi, and Y. Sawada, Diffusion and deformations of single hydra cells in cellular aggregates, Biophys. J, 79 (2000), pp. 1903-1914.

[127] J. Rubin And A. Robertson, The tip of the Dictyostelium pseudoplasmodium as an organizer, J. Embryol. Exp. Morphol., 33 (1975), pp. 227-241.

[128] B. Sager and D. Kaiser, Two cell-density domains within the Myxococcus xanthus fruiting body, Proc. Natl. Acad. Sci., 90 (1993), pp. 3690-3694.

[129] B. SAger AND D. KAISER, Intercellular C-signaling and the traveling waves of Myxococcus xanthus, Genes Development, 8 (1994), pp. 2793-2804.

[130] P. Sahni, G. Grest, M. Anderson, and D. Srolovitz, Kinetics of the Q-state Potts model in 2 dimensions, Phys. Rev. Lett., 50 (1983), pp. 263-266. D. Srolovitz, M. Anderson, G. Grest, and P. Sahni, Grain-growth in 2 dimensions, Scripta Met., 17 (1983), pp. 241-246. D. Srolovitz, M. Anderson, G. Grest, And P. SAhni, Computer-simulation of grain-growth. 2. Grain-size distribution, topology, and local dynamics, Acta Met., 32 (1984), pp. 793-802. D. Srolovitz, M. Anderson, G. Grest, and P. SAhni, Computer-simulation of grain-growth. 3. Influence of a particle dispersion, Acta Met., 32 (1984), pp. 1429-1438. G. Grest, D. Srolovitz, and M. Anderson, Kinetics of domain growth: universality of kinetic exponents, Phys. Rev. Letts,. 52 (1984), pp. 1321-1329. D. Srolovitz, G. Grest, and M. Anderson, Computersimulation of grain growth. 5. Abnormal grain-growth, Acta Met., 33 (1985), pp. 2233-2247.

[131] N. Savill and P. Hogeweg, Modelling morphogenesis: From single cells to crawling slugs, J. Theor. Bio., 184 (1997), pp. 229-235.

[132] M. Scalerandi, B. Sansone, and C. Condat, Diffusion with evolving sources and competing sinks: Development of angiogenesis, Phys. Rev. E, 65 (2002), 011902.

[133] J.A. Shapiro, Bacteria as multicellular organisms, Scientific American, 258 (1988), pp. 82-89.

[134] J.A. ShapIRo, The significances of bacterial colony patterns, Bioessays, 17 (1995), pp. 597-607.

[135] J.A. ShAPIRO, Thinking about bacterial populations as multicellular organisms, Annual Review of Microbiology, 52 (1998), pp. 81-104.

[136] N. Shimoyama, K. Sugawara, T. Mizuguchi, Y. Hayakawa, and M. Sano, Collective motion in a system of motile elements, Phys. Rev. Lett., 76 (1996), pp. $3870-3873$.

[137] E. Siggia, Late stages of spinodal decomposition in binary mixtures, Phys. Rev. A, 20 (1979), pp. 595-605.

[138] S. Simpson, A. McCaffery, and B. Hagele, A behavioural analysis of phase change in the desert locust, Bio. Rev. of the Cambridge Philosophical Society, 74 (1999), pp. 461-480.

[139] D. Soll, Computer-assisted three-dimensional reconstruction and motion analysis of living, crawling cells, Computerized Medical Imaging and Graphics, 23 (1999), pp. 3-14.

[140] D. Soll, E. Voss, O. Johnson, And D. Wessels, Three-dimensional reconstruction and motion analysis of living, crawling cells, Scanning, 22 (2000), pp. 249257.

[141] J. Stavans, The evolution of cellular structures, Rep. Prog. Phys., 56 (1993), pp. 733-789.

[142] M. Steinberg, Mechanism of tissue reconstruction by dissociated cells, II. Timecourse of events, Science, 137 (1962), pp. 762-763.

[143] M. Steinberg, Cell Membranes in Development, Academic Press, NY, 1964.

[144] A. Stevens, A stochastic cellular automaton modeling gliding and aggregation of Myxobacteria, SIAM J. Appl. Math., 61 (2000), pp. 172-182. 
[145] E. Stott, N. Britton, J. A. Glazier, and M. Zajac, Stochastic simulation of benign avascular tumour growth using the Potts model, Mathematical and Computer Modelling, 30 (1999), pp. 183-198.

[146] U. Technau and T. Holstein, Cell sorting during the regeneration of hydra from reaggregated cells, Devel. Bio, 151 (1992), pp. 117-127.

[147] D. Thompson, On Growth and Form, Cambridge University Press, Cambridge, 1942.

[148] A. Turing, The chemical basis of morphogenesis, Phil. Trans. R. Soc. London, 237 (1952), pp. 37-72.

[149] A. Upadhyaya, Thermodynamics and Fluid Properties of Cells, Tissues and Membranes, Ph.D. Thesis., The University of Notre Dame, USA, 2001.

[150] A. Upadhyaya, J. Rieu, J. A. Glazier and Y. Sawada, Anomalous diffusion and non-Gaussian velocity distribution of Hydra cells in cellular aggregates, Physica A, 293 (2001), pp. 49-558.

[151] P. Van HaAster, Sensory adaptation of Dictyostelium discoideum cells to chemotactic signals, J. Cell Biol., 96 (1983), pp. 1559-1565.

[152] B. Vasiev, F. Siegert and C.J. Weijer, A hydrodynamic model approach for Dictyostelium mound formation, J. Theor. Biol., 184 (1997), pp. 441-450.

[153] T. Vicsek, A. Czirok, E. Ben-Jacob, I Cohen, O. Shochet, and A. TenenBAum, Novel type of phase transition in a system of self-driven particles, Phys. Rev. Lett., 75 (1995), pp. 1226-1229.

[154] J. von Neumann, Theory of self-reproducing automata, (edited and completed by A. W. Burks), University of Illinois Press, Urbana, 1966.

[155] J. WartiovaAra, M. Karkinen-JäÄSkelänen, E. Lehtonen, S. Nordling, And L. SAxÉn, Morphogenetic Cell Interactions in Kidney Development, in N. Müller-Bér) (Ed.), Progress in Differentiation Research, North-Holland Publishing Company, Amsterdam, 1976, 245-252.

[156] D. Weaire and N. Rivier, Soap, cells and statistics: random patterns in 2 dimensions, Contemp. Phys. 25 (1984) pp. 59-99.

[157] H. Williams, S. Desjardins, and F. Billings, Two-dimensional growth models, Phys. Lett. A, 250 (1998), pp. 105-110.

[158] J. Williams, Regulation of cellular differentiation during Dictyostelium morphogenesis, Curr. Opin. Genet. Dev., 1 (1991), pp. 338-362.

[159] J. Wejchert, D. Weaire, and J. Kermode, Monte-Carlo simulation of the evolution of a two-dimensional soap froth, Phil. Mag. B, 53 (1986), pp. 15-24.

[160] R. Welch AND D. KAISER, Cell behavior in traveling wave patterns of myxobacteria, Proc. Natl. Acad. Sci. USA, 98 (2001), pp. 14907-14912.

[161] T. Witten And L. Sander, Diffusion-limited aggregation, Phys. Rev. B, 27 (1983), pp. 5686-5697.

[162] D. Wolf-Gladrow, Lattice-Gas Cellular Automata and Lattice Boltzmann Models - An Introduction, Springer-Verlag, Berlin, Lecture Notes in Mathematics 1725 (2000).

[163] S. Wolfram, Statistical mechanics of cellular automata, Rev. Mod. Phys., 55 (1983), pp. 601-604.

[164] S. Wolfram, Cellular Automata and Complexity, Addison-Wesley, Reading, 1994.

[165] S. Wolfram, A New Kind of Science, Wolfram Media, Champaign, 2002.

[166] C. Wolgemuth And E. HoiczyK, How Myxobacteria glide, Current Biology, 12 (2002), pp. 369-377.

[167] F. Wu, The Potts-model, Rev. Mod. Phys., 54 (1982), pp. 235-268.

[168] M. ZaJAC, G. Jones, AND J.A. Glazier, Model of convergent extension in animal morphogenesis, Phys. Rev. Lett., 85 (2000), pp. 2022-2025.

[169] M. ZaJac, Modeling Convergent Extension by Way of Anisotropic Differential Adhesion. Ph.D. thesis, The University of Notre Dame, USA, 2002. 